\title{
Markov and Stability Properties of Equilibrium States for Nearest-Neighbor Interactions
}

\author{
R. Kuik \\ Institute for Theoretical Physics, Postbox 800, NL-9700 AV Groningen, The Netherlands
}

\begin{abstract}
Consider models on the lattice $\mathbb{Z}^{d}$ with finite spin space per lattice point and nearest-neighbor interaction. Under the condition that the transfer matrix is invertible we use a transfer-matrix formalism to show that each Gibbs state is determined by its restriction to any pair of adjacent (hyper)planes. Thus we prove that (also in multiphase regions) translationally invariant states have a global Markov property. The transfer-matrix formalism permits us to view the correlation functions of a classical $d$-dimensional system as obtained by a linear functional on a noncommutative (quantum) system in $(d-1)$ dimensions. More precisely, for reflection positive classical states and an invertible transfer matrix the linear functional is a state. For such states there is a decomposition theory available implying statements on the ergodic decompositions of the classical state in $d$ dimensions. In this way we show stability properties of $\mathbb{Z}_{\mathrm{ev}}^{d}$-ergodic states and the absence of certain types of breaking of translational invariance.
\end{abstract}

\section{Introduction}

In this paper we study properties of and relations between the equilibrium states of some models with nearest-neighbor interactions on the lattice $\mathbb{Z}^{d}$. As a main tool we use a transfer-matrix formalism. This formalism enables us to prove a global Markov property with respect to (hyper)planes for invariant Gibbs states. This property entails that for such states the spins in $\left\{x \in \mathbb{Z}^{d} \mid x_{1}>0\right\}$ behave independently of the spins in $\left\{x \in \mathbb{Z}^{d} \mid x_{1}<0\right\}$ upon fixing the spins at the boundary $\left\{x \in \mathbb{Z}^{d} \mid x_{1}=0\right\}$.

Intuitively Markov properties and transfer-matrix techniques are closely related. The relation can be made explicit when considering a part of $\mathbb{Z}^{d}$, for instance $\bar{\Lambda}^{(N)} \equiv\left\{x \in \mathbb{Z}^{d}|| x_{i} \mid \leqq N\right.$ for $\left.i=1, \ldots, d-1\right\}$ with $N \in \mathbb{N}$, which is infinite only in one direction. In this case one is effectively considering a one-dimensional system. On all of $\mathbb{Z}^{d}$ however, the relation between the existence of finite-volume transfer matrices and Markov properties is not immediate, although if one assumes Markov properties the introduction of an infinite-volume transfer operator is not difficult, 
see e.g. [1]. In this paper we show how, under certain conditions, finite-volume transfer matrices can be used to derive Markov properties for invariant states.

Proving Markov properties requires some care. Indeed for the threedimensional Ising model Goldstein has presented an example of a Gibbs state that does not have a global Markov property [2], despite the existence of finite-volume transfer matrices. (This example involves a Gibbs state that is not translationally invariant.) When applying transfer-matrix techniques one usually starts out with finite-volume states and studies the Gibbs state(s) arising as thermodynamic-limit state(s) of these finite-volume states by means of the transfer-matrix formalism for the finite volumes.

We will develop a transfer-matrix formalism which can be applied directly to all Gibbs states. The important property we need is the invertibility of the transfer matrix. Let $q$ be the number of possible spin configurations per lattice point. Because we are assuming that only nearest-neighbor interaction is present, the invertibility of the transfer matrix is equivalent to the invertibility of a $q \times q$ matrix. Therefore checking the invertibility is relatively easy when considering specific models. Potts models (for nonzero coupling) have invertible transfer matrices and can therefore be handled by the methods in this paper. Note that the methods used are valid for arbitrary dimension $d$ of the lattice.

Let us sketch the transfer-matrix formalism that is used. Let $C_{0}(\Omega)$ be the space of functions on the configuration space $\Omega$ which depend on finitely many variables only. We construct a linear map $T$ from $C_{0}(\Omega)$ onto $a^{*}$-algebra $\mathfrak{A}_{0} \cdot \mathfrak{A}_{0}$ is dense in a $C^{*}$-algebra $\mathfrak{A}$. The map $T$ depends on the interaction. We show, if $\mu$ is a Gibbs state, that there exists a unique linear functional $l_{\mu}$ on $\mathfrak{P}_{0}$ such that

$$
\mu \uparrow_{C_{0}(\Omega)}=l_{\mu} \circ T \text {. }
$$

Stated as such this does not provide useful information on the Gibbs state $\mu$. Interesting results will be derived by studying the details of the relation between $C_{0}(\Omega)$ and $\mathfrak{U}_{0}$ which is given by the map $T$. For instance we show that every Gibbs state is uniquely determined by its restriction to two neighboring (hyper)planes: the expectation value of an observable in $C_{0}(\Omega)$ can be computed as the expectation value of another observable which depends only on variables in two (hyper)planes. This other observable depends on the interaction but not otherwise on the Gibbs state considered.

An important observation is that the map $T$ depends on $\Phi$ (and thus on the simplex of Gibbs states for $\Phi$ ) but not on the state $\mu$ in the set of Gibbs states individually. The map $T$ by transposition maps, affinely, the Gibbs states onto a set $L$ of linear functionals on $\mathfrak{A}_{0}$. Decompositions of invariant Gibbs states into other invariant Gibbs states using the noncommutative formalism will be used in the sequel of this paper to derive stability properties. However in case invariance is absent we are not able to use noncommutative decomposition theory since we lack a useful characterization of $L$.

In the construction of $T$ we make use of the maps $\mathfrak{U}_{0} \in \sigma \rightarrow V_{N} \sigma V_{N}^{-1} \in \mathfrak{A}_{0}$ for $N \in \mathbb{N}$ and where $V_{N}$ is the transfer matrix for the volume $\bar{\Lambda}^{(N)}$ in the unbounded direction. In particular the map $\alpha$ on $\mathfrak{A}_{0}$ which arises as the $N \rightarrow \infty$ limit of the above maps is used constructing $T$. This map $\alpha$ has been studied by Fredenhagen 
in the context of Euclidean lattice gauge theories to construct the real-time dynamics [3]. In fact the transfer-matrix method we use has a relation to Euclidean lattice field theories in case the Gibbs state $\mu$ is $\mathbb{Z}_{e v}^{d}$-invariant. It is shown that the state $\mu$ is reflection positive in this case. If a Gibbs state $\mu$ is $\mathbb{Z}_{e v}^{d}$-invariant the linear functional $l_{\mu}$ on $\mathfrak{U}_{0}$ associated with $\mu$ by (1) is a state. The Hilbert space constructed from reflection positivity of $\mu$, i.e., the physical Hilbert space, and the G.N.S. Hilbert space of $l_{\mu}$ are canonically isomorphic [3].

This paper can be considered as a continuation of a program which was started in a series of papers on the two-dimensional ferromagnetic Ising model. The state $l_{\mu}$, where $\mu$ is the Gibbs state with magnetization zero for this model has been obtained in [4 and 5]. The decomposition theory in the noncommutative setting was subsequently studied in [6 and 7]. These papers however did not emphasize the role of the relation (1), which plays a central role in this paper. In this way we rederive the main result of Araki and Evans on the decomposition of the state $l_{\mu},[7]$.

Before indulging into details let us make a further remark. Note that by change in representation a lattice system with a finite-range interaction can be viewed as a lattice system with next-nearest-neighbor interaction. For instance one can consider the array of spins in a (sufficiently large) block as a single spin variable.

Although the results in this paper could be largely extended if we were able to deal with interactions arising in such representations, we see two main difficulties. First for next-nearest-neighbor interactions the invertibility and locality properties, if true at all, are hard to establish. Such properties are needed in constructing $T$. Even if we could find a representation that has a nearest-neighbor interaction as for the axial-next-nearest-neighbor Ising model (ANNI-model), there remains a second difficulty. As the reader will realize, reflectional invariance is used often in this paper. If in the original, finite-range-interaction, representation of the lattice system the interaction is reflectionally invariant, this needs no longer be the case in the (next-)nearest-neighbor-interactions representation of the system, although, by transport of structure, in this last representation the same type of symmetry is still maintained. However this type of symmetry does not have the property of being associated with a plane of reflection, i.e., there is no (hyper)plane such that observables on this plane are invariant under the symmetry action. It is precisely such a property, for reflections, that is used in the proofs of Theorem 2 and Lemma 2 of this paper and which are basic to the results obtained. Therefore we think that generalizations along this line of results in Theorem 2 and Lemma 2 are not immediate.

Moreover straightforward generalizations along this line of all the results seem impossible in view of the results on the ANNI-model in [16] compared to those in Corollary 4.

\section{Definitions}

Let $\Omega_{0}$ be the finite set $\{1, \ldots, q\} \subset \mathbb{N}$. We equip $\Omega_{0}$ with the discrete topology. For $\Lambda \subseteq \mathbb{Z}^{d}$ we put $\Omega_{\Lambda}=\left(\Omega_{0}\right)^{\Lambda}$. It is assumed that $d \geqq 2$. Write $\Omega_{\mathbb{Z}^{d}}=\Omega$. We equip $\Omega_{\Lambda}$ with the product topology and denote by $C\left(\Omega_{\Lambda}\right)$ the space of continuous functions on $\Omega_{\Lambda^{\prime}}$. Let $\alpha_{\Lambda} \Lambda^{\prime}: \Omega_{\Lambda^{\prime}} \rightarrow \Omega_{\Lambda}$ be the restriction map if $\Lambda^{\prime} \supset \Lambda$. We write 
$\alpha_{\Lambda, \mathbb{Z}^{d}}=\alpha_{\Lambda}$. Define $C_{\Lambda}=\left\{f \in C(\Omega) \mid\right.$ there exists $f_{\Lambda} \in C\left(\Omega_{\Lambda}\right)$ such that $\left.f=f_{\Lambda} \circ \alpha_{\Lambda}\right\}$. The map $C\left(\Omega_{\Lambda}\right) \in f_{\Lambda} \rightarrow f_{\Lambda} \circ \alpha_{\Lambda} \in C_{\Lambda}$ constitutes an isomorphism of $C\left(\Omega_{\Lambda}\right)$ onto $C_{\Lambda}$. Henceforth we identify $C\left(\Omega_{\Lambda}\right)$ and $C_{\Lambda}$ by means of this isomorphism.

For $\Lambda \subseteq \mathbb{Z}^{d}$ we denote by $\mathfrak{B}_{\Lambda}$ the $\sigma$-algebra generated by the sets

$$
\left\{\xi \in \Omega \mid \alpha_{\Lambda^{\prime}}(\xi)=\xi_{\Lambda^{\prime}}\right\}_{\substack{\Lambda^{\prime} \subseteq \Lambda \\ \Lambda^{\prime} \text { finte } \\ \xi_{\Lambda^{\prime} \in \Omega_{\Lambda^{\prime}}}}} .
$$

We write $\mathfrak{B}_{\mathbb{Z}^{d}}=\mathfrak{B}$. Furthermore we let $\mathfrak{B}\left(\Omega_{\Lambda}\right)$ denote the Borel $\sigma$-algebra on $\Omega_{\Lambda}$. For finite $\Lambda^{\prime} \subseteq \Lambda$ and $\xi_{\Lambda^{\prime}} \in \Omega_{\Lambda^{\prime}}$ consider the map

$$
\mathfrak{B}\left(\Omega_{\Lambda}\right) \ni\left\{\xi \in \Omega_{\Lambda} \mid \alpha_{\Lambda^{\prime}, \Lambda}(\xi)=\xi_{\Lambda^{\prime}}\right\} \rightarrow\left\{\xi \in \Omega \mid \alpha_{\Lambda^{\prime}}(\xi)=\xi_{\Lambda^{\prime}}\right\} \in \mathfrak{B}_{\Lambda^{\prime}}
$$

This map, for all finite $\Lambda^{\prime} \subseteq \Lambda$ and $\xi_{\Lambda^{\prime}} \in \Omega_{\Lambda^{\prime}}$, constitutes an isomorphism of the algebras $\mathfrak{B}\left(\Omega_{\Lambda}\right)$ and $\mathfrak{B}_{\Lambda}$. Henceforth we identify $\mathfrak{B}\left(\Omega_{\Lambda}\right)$ and $\mathfrak{B}_{\Lambda}$ by means of this isomorphism. We denote by $E_{C\left(\Omega_{\Lambda}\right)}$ the set of states on $C\left(\Omega_{\Lambda}\right) . E_{C\left(\Omega_{N}\right)}$ is equipped with the weak * topology. A state $\mu$ on $C\left(\Omega_{\Lambda}\right)$ is identified with a (Borel) probability measure $d \mu$ or $\mathfrak{B}\left(\Omega_{\Lambda}\right)$ by

$$
\mu(f)=\int_{\Omega_{\Lambda}} f d \mu \text { for } f \in C\left(\Omega_{\Lambda}\right) .
$$

If $\mu$ is a probability measure on $\mathfrak{B}_{\Lambda}$ we denote by $\mathbb{E}_{\Lambda^{\prime}}^{\mu}$, if $\Lambda^{\prime} \subset \Lambda$, the orthogonal projection acting on $L^{2}\left(\mu ; \mathfrak{B}_{\Lambda}\right)$ with range $L^{2}\left(\mu ; \mathfrak{B}_{\Lambda^{\prime}}\right)$. Let $\Phi: \bigcup_{\substack{\Lambda \text { finite } \\ \Lambda \subset \mathbb{Z}^{d}}} \Omega_{\Lambda} \rightarrow \mathbb{R}$ be a nearest-neighbor potential: $\Phi \circ \alpha_{\Lambda} \equiv 0$ unless $\Lambda$ is a subset of a nearest-neighbor pair. The Hamiltonian, $H_{\Lambda}^{\Phi}$ for a finite volume $\Lambda \subset \mathbb{Z}^{d}$ is a function in $C_{\Lambda}$ defined by

$$
H_{\Lambda}^{\Phi}=\sum_{X \subset \Lambda} \Phi \circ \alpha_{X}
$$

Furthermore, if $\Lambda$ is finite, we define $W_{\Lambda}^{\Phi} \in C$ by

$$
W_{\Lambda}^{\Phi}=\sum_{\substack{X \cdot X \cap A \neq \phi \\ X \cap \Lambda^{c} \neq \phi}} \Phi \circ \alpha_{X}
$$

More generally, for $\Lambda$ finite and $\Lambda^{\prime} \subseteq \mathbb{Z}^{d}$, we define $W_{\Lambda \cdot \Lambda^{\prime}} \in C_{\Lambda \cup \Lambda^{\prime}}$, by

$$
W_{\Lambda, \Lambda^{\prime}}=\sum_{\substack{\mathrm{X} \cdot \mathrm{X} \cap \Lambda \neq \phi \\ X \cap \Lambda \neq \phi \\ X \subseteq \Lambda \cup \Lambda^{\prime}}} \Phi \circ \alpha_{X}
$$

For $\Lambda, \Lambda \subset \mathbb{Z}^{d}$ with $\Lambda \cap \Lambda^{\prime}=\phi$ we define, if $\xi_{\Lambda} \in \Omega_{\Lambda}$ and $\xi_{\Lambda^{\prime}} \in \Omega_{\Lambda^{\prime}}$, the configuration $\xi_{\Lambda} \vee \xi_{\Lambda^{\prime}} \in \Omega_{\Lambda \cup \Lambda^{\prime}}$ by

$$
\alpha_{\Lambda, \Lambda \cup \Lambda^{\prime}}\left(\xi_{\Lambda} \vee \xi_{\Lambda^{\prime}}\right)=\xi_{\Lambda} \quad \text { and } \quad \alpha_{\Lambda^{\prime}, \Lambda \cup \Lambda^{\prime}}\left(\xi_{\Lambda} \vee \xi_{\Lambda^{\prime}}\right)=\xi_{\Lambda^{\prime}}
$$

Define for finite $\Lambda \subset \mathbb{Z}^{d}$ and $\xi_{\Lambda^{c}} \in \Omega_{\Lambda^{c}}$ the function $\rho_{(\Lambda), \xi_{\Lambda^{c}}}^{\Phi} \in C_{\Lambda}$ by

$$
\rho_{(\Lambda), \xi_{\Lambda^{c}}}^{\Phi}\left(\xi_{\Lambda}\right)=\frac{\exp \left[-\left(H_{\Lambda}^{\Phi}\left(\xi_{\Lambda}\right)+W_{\Lambda}^{\Phi}\left(\xi_{\Lambda} \vee \xi_{\Lambda^{c}}\right)\right)\right]}{Z_{(\Lambda), \xi_{\Lambda^{c}}}^{\Phi}}
$$

where $Z_{(\Lambda), \zeta_{A}}^{\Phi}$ is determined by the normalization

$$
\sum_{\xi_{\Lambda} \in \Omega_{\Lambda}} \rho_{(\Lambda), \zeta_{\Lambda} c}^{\Phi}\left(\xi_{\Lambda}\right)=1
$$


For finite $\Lambda \subset \mathbb{Z}^{d}$ we introduce the operator $\mathbb{E}_{\Lambda^{c}}: C(\Omega) \rightarrow C(\Omega)$ by

$$
\left(\mathbb{E}_{\Lambda^{c}}^{\Phi} f\right)(\xi)=\sum_{\xi_{\Lambda^{\prime}} \in \Omega_{\Lambda}} f\left(\xi_{\Lambda} \vee \alpha_{\Lambda^{c}}(\xi)\right) \rho_{(\Lambda), \alpha_{\Lambda^{c}}(\xi)}^{\Phi}\left(\xi_{\Lambda}\right),
$$

where $f \in C(\Omega)$ and $\left(\mathbb{E}_{\Lambda^{c}}^{\Phi} f\right)(\xi)$ is the value of the function $\mathbb{E}_{\Lambda^{c}}^{\Phi} f$, which is the image of $f$ under $\mathbb{E}_{\Lambda^{c}}^{\Phi}$, at the point $\xi \in \Omega$. A probability measure $\mu$ on $\mathfrak{B}$ is said to be a Gibbs state for the interaction $\Phi$ if $\mu(f)=\mu\left(\mathbb{E}_{\Lambda^{c}}^{\Phi}(f)\right)$ for all $f \in C(\Omega)$ and finite $\Lambda \subset \mathbb{Z}^{d}$. The set of Gibbs states for $\Phi$ is denoted by $\mathscr{G}_{\Phi}$.

Next we introduce the noncommutative *algebras that we need. Let $\left(\mathbb{C}^{q}\right)_{y}$ be a copy of $\mathbb{C}^{q}$ for each $y \in \mathbb{Z}^{d-1}$. We number the coordinate axes of $\mathbb{Z}^{d-1}$ from 1 to $d-1$. Let $\mathfrak{A}$ be the $C^{*}$-algebra defined as

$$
\mathfrak{U}=\bigotimes_{y \in \mathbb{Z}^{d-1}} \mathfrak{L}\left(\left(\mathbb{C}^{q}\right)_{y}\right)
$$

where $\mathfrak{L}\left(\left(\mathbb{C}^{q}\right)_{y}\right)$ is the algebra of linear operators on $\left(\mathbb{C}^{q}\right)_{y}$. More generally we set

$$
\mathfrak{U}_{\tilde{\Lambda}}=\bigotimes_{y \in \tilde{\Lambda}} \mathfrak{L}\left(\left(\mathbb{C}^{q}\right)_{y}\right)
$$

if $\tilde{\Lambda} \subset \mathbb{Z}^{d-1}$. The $C^{*}$-algebra $\mathfrak{U}_{\tilde{\Lambda}}$ is considered as a subalgebra of $\mathfrak{U}$. We define $\mathfrak{U}_{0}$ as the *subalgebra given by

$$
\mathfrak{U}_{0}=\bigcup_{\substack{\tilde{\Lambda} \text { finite } \\ \tilde{\Lambda} \subset \mathbb{Z}^{d-1}}} \mathfrak{A}_{\tilde{\Lambda}}
$$

Define the Hilbert space $\mathfrak{H}_{e}^{\otimes}$ as $\mathfrak{H}_{e}^{\otimes}=\bigotimes_{y \in \mathbb{Z}^{d-1}}\left(\mathbb{C}^{q}\right)_{y}$ associated with the vector $\Omega_{e}=\bigotimes_{y \in \mathbb{Z}^{d-1}} e_{y} \in \mathfrak{H}_{e}^{\otimes}$, where $e_{y} \in\left(\mathbb{C}^{q}\right)_{y}$ is the unit vector obtained from a unit vector $e \in \mathbb{C}^{q}$ by the process of taking the copy $\left(\mathbb{C}^{q}\right)_{y}$ of $\mathbb{C}^{q}$. Define $\pi_{e}$ as the * representation of $\mathfrak{A}$ on $\mathfrak{H}_{e}^{\otimes}$, which satisfies for each $\tilde{y} \in \mathbb{Z}^{d-1}$,

$$
\pi_{e}(\sigma) \bigotimes_{y \in \mathbb{Z}^{d-1}} e_{y}^{\prime}=\sigma e_{\tilde{y}}^{\prime} \otimes\left(\bigotimes_{y \in \mathbb{Z}^{d-1} \backslash\{\tilde{y}\}} e_{y}^{\prime}\right)
$$

for all $\sigma \in \mathfrak{A}_{\{\hat{y}\}} \subset \mathfrak{U}$ and all choices of unit vectors $e_{y}^{\prime} \in\left(\mathbb{C}^{q}\right)_{y}$ such that $e_{y}^{\prime} \neq e_{y}$ only for finitely many $y \in \mathbb{Z}^{d-1}$.

Let $\tilde{\Lambda} \subset \mathbb{Z}^{d-1}$ be finite and $\eta_{\tilde{\Lambda}} \in\left(\Omega_{0}\right)^{\tilde{\Lambda}}$. Then we define $\tilde{\eta}_{\tilde{\Lambda}} \in \mathfrak{H}_{e}^{\otimes}$ by

$$
\tilde{\eta}_{\tilde{\Lambda}}=\bigotimes_{y \in \mathbb{Z}^{d-1}} \tilde{e}_{y}
$$

where

$$
\tilde{e}_{y}=\left\{\begin{array}{lll}
\left(e_{(\eta \tilde{\Lambda})_{y}}\right)_{y} & \text { for } & y \in \tilde{\Lambda} \\
e_{y} & \text { for } & y \in \tilde{\Lambda}^{c} .
\end{array}\right.
$$

Here $\left(\eta_{\tilde{\Lambda}}\right)_{y}$ is the value of the configuration $\eta_{\tilde{\Lambda}}$ at $y \in \tilde{\Lambda}$ and $\left(e_{l}\right)_{y}$ is the $l$-th element of the standard basis $\left\{e_{1}, \ldots, e_{q}\right\}$ of $\mathbb{C}^{q}$ under the process of taking the copy $\left(\mathbb{C}^{q}\right)_{y}$ of $\mathbb{C}^{q}$.

Let $M^{(\tilde{\Lambda})}$ be a $q^{|\tilde{\Lambda}|} \times q^{|\tilde{\Lambda}|}$ matrix with matrix elements

$$
\left\{M_{\eta_{\tilde{\Lambda}}^{\tilde{n}^{n} \tilde{\Lambda}^{\prime}}}^{(\tilde{\tilde{\Lambda}})}\right\}_{\substack{\eta_{\tilde{\Lambda}} \in\left(\Omega_{0}\right) \\ \eta_{\tilde{\Lambda}}^{\prime} \in\left(\Omega_{0}\right)^{\tilde{\Lambda}}}}
$$


for finite $\tilde{\Lambda} \subset \mathbb{Z}^{d-1}$. We also denote by $M^{(\tilde{\Lambda})}$ the element of $\mathfrak{A}_{\tilde{\Lambda}} \subset \mathfrak{A}_{0} \subset \mathfrak{H}$ which satisfies

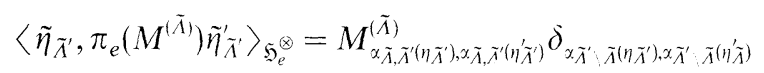

for all $\eta_{\tilde{\Lambda}^{\prime}}, \eta_{\tilde{\Lambda}^{\prime}}^{\prime} \in\left(\Omega_{0}\right)^{\tilde{\Lambda}^{\prime}}$, where $\tilde{\Lambda}^{\prime} \supseteq \tilde{\Lambda}$. Here $\langle\cdot, \cdot\rangle_{\mathfrak{S}_{e}^{\otimes}}$ is the inner product of $\mathfrak{H}_{e}^{\otimes}$ and $\alpha_{\tilde{\Lambda}, \tilde{\Lambda}^{\prime}}$ is the operation of restricting configurations on $\tilde{\Lambda}^{\prime} \subset \mathbb{Z}^{d-1}$ to $\tilde{\Lambda} \subset \mathbb{Z}^{d-1}$, analogously to the restriction operator we introduced for subsets of $\mathbb{Z}^{d}$.

The operator $M^{(\tilde{\Lambda})} \in \mathfrak{A}_{\tilde{\Lambda}}$ is well-defined by (2) and does not depend on $e \in \mathbb{C}^{q}$. We denote by $\mathfrak{I}_{0}^{\text {diag }}$ the sub*-algebra of $\mathfrak{A}_{0}$ which consists of operators in $\mathfrak{U}_{0}$ that can be defined by a diagonal matrix in (2). The algebra $\mathfrak{A}_{0}^{\text {diag }}$ is commutative. Write $\mathfrak{U}_{\tilde{\Lambda}}^{\text {diag }}=\mathfrak{U}_{0}^{\text {diag }} \cap \mathfrak{U}_{\tilde{\Lambda}}$. The set of states on $\mathfrak{A}$ is denoted by $E_{\mathfrak{Q}} . E_{\mathfrak{Q}}$ is equipped with the weak* topology.

\section{Formalism}

For $\Lambda \subset \mathbb{Z}^{d}$, define $\partial \Lambda=\left\{x \in \mathbb{Z}^{d} \mid d(x, \Lambda)=1\right\}$, where $d(\cdot, \cdot)$ is the standard metric on $\mathbb{Z}^{d} \times \mathbb{Z}^{d} \subset \mathbb{R}^{d} \times \mathbb{R}^{d}$. Furthermore, for $n, k \in \mathbb{N}$, define

$$
P_{k}(n)=\left\{x \in \mathbb{Z}^{d}\left|x_{0}=k ; \quad\right| x_{i} \mid \leqq n \quad \text { for } \quad i=1, \ldots, d-1\right\}
$$

and

$$
P(n)=\left\{y \in \mathbb{Z}^{d-1}|| y_{i} \mid \leqq n \text { for } i=1, \ldots, d-1\right\} .
$$

Put $R_{k}(n)=\left(\Lambda_{n+1} \backslash \Lambda_{n}\right) \cap P_{k}(n+1)$, where $\Lambda_{n} \subset \mathbb{Z}^{d}$ is for $n \in \mathbb{N}$ given by

$$
\Lambda_{n}=\left\{x \in \mathbb{Z}^{d}|| x_{i} \mid \leqq n \text { for } i=0, \ldots, d-1\right\} .
$$

Choose $\xi_{n} \in \Omega_{\Lambda_{n}^{c}} ; n \in \mathbb{N}$. Define $V_{k ; n, \perp}\left(\xi_{n}\right) \in \mathfrak{A}_{0}$ by means of its matrix elements defined by

$$
\left(V_{k ; n, \perp}\left(\xi_{n}\right)\right)_{\eta, \eta^{\prime}}=\delta_{\eta, \eta^{\prime}} \exp \left[-H_{P_{k}(n+1)}^{\Phi}\left(j_{k} \eta \vee \alpha_{R_{k}(n)} \xi_{n}\right)\right],
$$

where $\eta, \eta^{\prime} \in\left(\Omega_{0}\right)^{P(n)}$ and $j_{k} \eta \in\left(\Omega_{0}\right)^{P_{k}(n)}$ is, for $\eta \in\left(\Omega_{0}\right)^{P(n)}$, defined by

$$
\left(j_{k} \eta\right)_{\left(k, y_{1}, \ldots, y_{d-1}\right)}=\eta_{\left(y_{1}, \ldots, y_{d-1}\right)}
$$

with $y=\left(y_{1}, \ldots, y_{d-1}\right) \in P(n) \subset \mathbb{Z}^{d-1}$. Furthermore define $V_{k+1 / 2, n, 0} \in \mathfrak{P}_{0}$ by means of its matrix elements defined by

$$
\left(V_{k+1 / 2 ; n, 0}\right)_{\eta, \eta^{\prime}}=\exp \left[-W_{P_{k}(n), P_{k+1}(n)}^{\Phi}\left(j_{k} \eta \vee j_{k+1} \eta^{\prime}\right)\right] .
$$

Consider a function $f \in C_{\Lambda_{n}}$ of the form

$$
f=f_{-l} \cdots f_{l},
$$

where $f_{k} \in C_{p_{k}(n)}$ for $k=-l, \ldots, 1$ and where $l \in \mathbb{N}$. In the standard plane to plane transfer-matrix formalism one writes

$$
\begin{aligned}
\left(\mathbb{E}_{\Lambda_{n}^{c}}^{\Phi} f\right)\left(\xi_{n}\right)= & \frac{1}{Z_{\left(\Lambda_{n}\right), \xi}^{\Phi}}\left\langle\left(j_{-(n+1)}^{-1} \circ \alpha_{P_{-(n+1)}(n)} \xi_{n}\right)^{\wedge}, \pi_{e}\left(\left(V_{-n . n, \perp}\left(\xi_{n}\right)\right)^{1 / 2}\right.\right. \\
& \cdot V_{-n+1 / 2, n}\left(\xi_{n}\right) \cdots V_{-\left(l+1^{1 / 2}\right) ; n}\left(\xi_{n}\right) V_{-(l+1 / 2), n}\left(\xi_{n}\right) \hat{f}_{-l} V_{-1+1 / 2 ; n}\left(\xi_{n}\right)
\end{aligned}
$$




$$
\begin{aligned}
& \cdot \hat{f}_{-l+1} V_{-l+1^{1 / 2} ; n}\left(\xi_{n}\right) \hat{f}_{-l+2} \cdots V_{l-1^{1 / 2} ; n}\left(\xi_{n}\right) \hat{f}_{l-1} V_{l-1 ; n}\left(\xi_{n}\right) \hat{f}_{l} V_{l+1 / 2 ; n}\left(\xi_{n}\right) \\
& \left.\left.\cdot V_{l+1^{1 / 2}: n}\left(\xi_{n}\right) \cdots V_{n-1 / 2 ; n}\left(\xi_{n}\right)\left(V_{n ; n, 2}\left(\xi_{n}\right)\right)^{1 / 2}\right)\left(j_{(n+1)^{\circ}}^{\circ} \alpha_{P_{n+1}(n)} \xi_{n}\right)^{\wedge}\right\rangle_{\mathfrak{S}_{e}^{\otimes}},
\end{aligned}
$$

where

$$
V_{k+1 / 2 ; n}\left(\xi_{n}\right)=\left(V_{k ; n, \perp}\left(\xi_{n}\right)\right)^{1 / 2} V_{k+1 / 2 ; n, 0}\left(V_{k+1 ; n, \perp}\left(\xi_{n}\right)\right)^{1 / 2}
$$

and $\widehat{f}_{k} \in \mathfrak{A}_{0}^{\text {diag }}$ is for $k=-l, \ldots, l$ defined by its matrix elements given by

$$
\left(\tilde{f}_{k}\right)_{\eta, \eta^{\prime}}=\delta_{\eta, \eta^{\prime}} f_{k}\left(j_{k} \eta\right)
$$

for $\eta, \eta^{\prime} \in\left(\Omega_{0}\right)^{P(m)}$ with $n \leqq m \in \mathbb{N}$. The operators $V_{k ; n, \perp}\left(\xi_{n}\right)$ and $V_{k+1 / 2 ; n, 0}$ can be expressed as products of more elementary operators. Let $[y, z]$ denote a nearestneighbor pair of points $y, z \in \mathbb{Z}^{d-1}$ (with nearest measured by the standard metric on $\left.\mathbb{Z}^{d-1} \times \mathbb{Z}^{d-1} \subset \mathbb{R}^{d-1} \times \mathbb{R}^{d-1}\right)$ ordered such that $(z-y)_{i}=0,1$ for $i=1, \ldots, d-1$. The set of such ordered nearest-neighbor pairs in $P(n) \subset \mathbb{Z}^{d-1}$ is denoted by $\widetilde{P}_{n}$. Then there exist operators $v_{k, \perp}([y, z]) \in \mathfrak{A}_{\{, z\}}$ for a nearest-neighbor pair $[y, z]$ in $\mathbb{Z}^{d-1}, w_{k}(y) \in \mathfrak{U}_{\{y\}}$ for $y \in \mathbb{Z}^{d-1}$ and $\omega_{k}^{\xi_{n}}(y) \in \mathfrak{U}_{\{y\}}$ for $y \in \partial \Lambda_{n}^{c}$ such that

$$
V_{k, n, \perp}\left(\xi_{n}\right)=\left(\prod_{[y, z] \in \tilde{P}_{n}} v_{k, \perp}([y, z])\right)\left(\prod_{y \in \Lambda_{n}} \omega_{k}(y)\right)\left(\prod_{y \in \hat{o} \Lambda_{n}^{c}} \omega_{k}^{\xi_{n}}(y)\right) .
$$

The operators appearing in the above product form of $V_{k, n, \perp}$ are defined by means of their matrix elements which are given by

$$
\left(v_{k, \perp}([y, z])\right)_{\eta, \eta^{\prime}}=\delta_{\eta, \eta^{\prime}} \exp -W_{\left\{j_{k} y\right\},\left\{j_{k} z^{z}\right.}^{\Phi}\left(j_{k} \eta\right)
$$

for $\eta, \eta^{\prime} \in\left(\Omega_{0}\right)^{\{y, z\}}$ and $[y, z]$ a nearest-neighbor pair,

$$
\left(w_{k}(y)\right)_{\eta, \eta^{\prime}}=\delta_{\eta, \eta^{\prime}} \exp \left[-H_{\left\{i_{k} y\right\}}^{\Phi}\left(j_{k} \eta\right)\right]
$$

for $\eta, \eta^{\prime} \in\left(\Omega_{0}\right)^{\{y\}}$ and $y \in \mathbb{Z}^{d-1}$, and

$$
\left(w_{k}^{\xi_{n}}(y)\right)_{\eta, \eta^{\prime}}=\delta_{\eta, \eta^{\prime}} \exp -W_{\left\{J_{k} y\right\},\left\{x_{y}\right\}}^{\Phi}\left(j_{k} \eta \vee \alpha_{\left\{x_{y}\right\}} \xi_{n}\right)
$$

for $\eta, \eta^{\prime} \in\left(\Omega_{0}\right)^{\{y\}}$ and $y \in \partial \Lambda_{n}^{c}$, and where $x_{y} \in \partial \Lambda_{n}$ is defined by $d\left(j_{k} y, x_{y}\right)=1$. The following definitions were used. If $y \in \mathbb{Z}^{d-1}$ then $j_{k} y \in \mathbb{Z}^{d}$ is given as

$$
j_{k} y=\left(k, y_{1}, \ldots, y_{d-1}\right) \in \mathbb{Z}^{d}
$$

and if $\eta \in \Omega_{0}^{\tilde{\Lambda}}$ for $\tilde{\Lambda} \subset \mathbb{Z}^{d-1}$, then $j_{k} \eta \in\left(\Omega_{0}\right)^{J_{k} \tilde{\Lambda}}$ is defined as $\left(j_{k} \eta\right)_{\left(k, y_{1}, \ldots, y_{d-1}\right)}=\eta_{\left(y_{1}, \ldots, y_{d-1}\right)}$ for $y \in \tilde{\Lambda}$. Note that all the operators that were just introduced are in $\mathfrak{A}_{0}^{\text {diag }}$. Define furthermore the contact operators $v_{k+1 / 2,0}(y) \in \mathfrak{U}_{\{y\}}$ by their matrix elements given by

$$
\left(v_{k+1 / 2,0}(y)\right)_{\eta, \eta^{\prime}}=\exp -W_{\left\{J_{k} y\right\},\left\{j_{k+1} y\right\}}^{\Phi}\left(j_{k} \eta \vee j_{k+1} \eta^{\prime}\right)
$$

for $\eta, \eta^{\prime} \in\left(\Omega_{0}\right)^{\{y\}}$. Then $V_{k+1 / 2: n, 0}=\prod_{y \in P(n)} v_{k+1 / 2,0}(y)$. Henceforth the following condition, Condition $C_{1}$, is assumed to hold for the potential $\Phi$.

Condition $C_{1}$. For all $k \in \mathbb{Z}$ and $y \in \mathbb{Z}^{d-1}$ the contact operators $v_{k+1 / 2,0}(y)$ are invertible.

Condition $C_{1}$ implies that the operators $V_{k+1 / 2 ; n, 0}$ are invertible for $n \in \mathbb{N}, k \in \mathbb{Z}$. 
Hence the operators $V_{k+1 / 2 ; n}\left(\xi_{n}\right)$ are invertible for each choice of $\xi_{n} \in \Omega_{\Lambda_{n}^{c}}$. Define the map $\alpha^{(k) ; ; \xi_{n}}: \mathfrak{A}_{0} \rightarrow \mathfrak{A U}_{0}$ which maps $\sigma \in \mathfrak{U}_{0}$ onto $\alpha^{(k), n ; \xi_{n}}(\sigma) \in \mathfrak{A}_{0}$ by:

1) if $k>0$, then

$$
\alpha^{(k) ; n, \xi_{n}}(\sigma)=V_{1 / 2 ; n}\left(\xi_{n}\right) \cdots V_{k-1 / 2, n}\left(\xi_{n}\right) \sigma\left(V_{k-1 / 2, n}\left(\xi_{n}\right)\right)^{-1} \cdots\left(V_{1 / 2 ; n}\left(\xi_{n}\right)\right)^{-1} ;
$$

2) if $k=0$, then $\alpha^{(k) ; ; \xi_{n}}$ is the identity on $\mathfrak{A}_{0}$;

3) if $k<0$, then

$$
\alpha^{(k) ;, \xi_{n}}(\sigma)=\left(V_{-1 / 2, n}\left(\xi_{n}\right)\right)^{-1} \cdots\left(V_{k+1 / 2, n}\left(\xi_{n}\right)\right)^{-1} \sigma V_{k+1 / 2 ; n}\left(\xi_{n}\right) \cdots V_{-1 / 2 ; n}\left(\xi_{n}\right) .
$$

Observing that $\left[\sigma_{\tilde{\Lambda}}, \sigma_{\tilde{\Lambda}^{\prime}}\right]_{-}=0$ if $\sigma_{\tilde{\Lambda}} \in \mathfrak{U}_{\tilde{\Lambda}}$ and $\sigma_{\tilde{\Lambda}^{\prime}} \in \mathfrak{H}_{\tilde{\Lambda}^{\prime}}$ with $\tilde{\Lambda} \cap \tilde{\Lambda}^{\prime}=\phi$, it readily follows that for $\sigma \in \mathfrak{A}_{0}$ there exist $n_{0} \in \mathbb{N}$ such that the elements in the sequence $\left\{\alpha^{(k) ; n ; \xi_{n}}(\sigma)\right\}_{\substack{n \geqq n_{0} \\ n \in \mathbb{N}}}$ do not depend on $n$ anymore, nor on the configurations $\xi_{n} \in \Omega_{\partial A_{n}}$.

Thus one can define for $k \in \mathbb{Z}$ the map $\alpha^{(k)}: \mathfrak{U}_{0} \rightarrow \mathfrak{A}_{0}$ which maps $\sigma \in \mathfrak{A}_{0}$ onto $\alpha^{(k)}(\sigma) \in \mathfrak{A}_{0}$ by

$$
\alpha^{(k)}(\sigma)=\lim _{n \rightarrow \infty} \alpha^{(k), n, \breve{\zeta}_{n}}(\sigma)
$$

Define

$$
V_{k ; n, \perp}=\left(\prod_{[y, z] \in \tilde{P}_{n}} v_{k, \perp}([y, z])\right)\left(\prod_{y \in \Lambda_{n}} w_{k}(y)\right)
$$

and

$$
V_{k+1 / 2, n}=\left(V_{k, n, \perp}\right)^{1 / 2} V_{k+1 / 2, n, 0}\left(V_{k+1, n, \perp}\right)^{1 / 2} .
$$

Define the map $\alpha_{(k) ; n}: \mathfrak{U}_{0} \rightarrow \mathfrak{A U}_{0}$ by the same formulas as used when defining $\alpha^{(k) ; n ; \xi_{n}}$ but under substituting $V_{k, n}$ for $V_{k ; n}\left(\xi_{n}\right)$. Then, if $\sigma \in \mathfrak{A}_{\tilde{\Lambda}}$ for a finite $\widetilde{\Lambda} \subset \mathbb{Z}^{d-1}$,

$$
\alpha^{(k)}(\sigma)=\lim _{n \rightarrow \infty} \alpha^{(k) ; n}(\sigma),
$$

which shows the independence of $\alpha^{(k)}(\sigma)$ on $\left\{\xi_{n}\right\}_{n \in \mathbb{Z}}$ explicitly. Moreover if $\tilde{\Lambda} \subset \tilde{\Lambda}_{n} \equiv\left\{y \in \mathbb{Z}^{d-1}|| y_{i} \mid \leqq n\right.$ for $\left.i=1, \ldots, d-1\right\}$, then for $k>0$ and $\sigma \in \mathfrak{U}_{\tilde{\Lambda}}$, $\alpha^{(k)}(\sigma)=V_{1 / 2 ; n+2 k} V_{1^{1 / 2} ; n+2(k-1)} \cdots V_{k-1 / 2, n+2} \sigma\left(V_{k-1 / 2 ; n+2}\right)^{-1} \cdots\left(V_{1^{1 / 2}: n+2(k-1)}\right)^{-1}\left(V_{1 / 2, n+2 k}\right)^{-1}$.

With a similar formula for the case $k<0$ it is readily seen, by using $V_{k+1 / 2, n}=$ $\left(V_{k, n, \perp}\right)^{1 / 2} V_{k+1 / 2 ; n, 0}\left(V_{k+1, n, \perp}\right)^{1 / 2}$ that $\alpha^{(k)}(\sigma) \in \mathfrak{U}_{\tilde{\Lambda}_{n+|k|+1}}$ when $\sigma \in \mathfrak{U}_{\tilde{\Lambda}_{n}}$. Define $T^{n, \xi_{n}}$ : $C_{0}(\Omega) \rightarrow \mathfrak{U}_{0}$ for $n \in \mathbb{N}$ and $\xi_{n} \in \Omega_{\Lambda_{n}^{c}}$ as the unique linear map that maps $f \in C_{0}(\Omega)$ onto $T^{n, \xi_{n}}(f) \in \mathfrak{A}_{0}$, where $T^{n, \xi_{n}}(f)$ is given by

$$
T^{n, \xi_{n}}(f)=\alpha^{\left(-l, n, \xi_{n}\right.}\left(\hat{f}_{-l}\right) \cdots \alpha^{(l) ; n ; \xi_{n}}\left(\hat{f}_{l}\right)
$$

for $f$ of the form $f=f_{-l} \cdots f_{l}$ with $f_{k} \in \bigcup_{n=1}^{\infty} C_{P_{k}(n)}$ for $k=-l, \ldots, l$. The map $T^{n, \zeta_{n}}$ is well-defined. Then

$$
\left(\mathbb{E}_{\Lambda_{n}^{c}} f\right)\left(\xi_{n}\right)=\frac{1}{Z_{\left(\Lambda_{n}\right), \xi_{n}}^{\Phi}}\left\langle\left(j_{-(n+1)}^{-1} \circ \alpha_{P_{-(n+1)}(n)} \xi_{n}\right)^{\wedge},\right.
$$




$$
\begin{aligned}
& \cdot \pi_{e}\left(\left(V_{-n ; n}\left(\xi_{n}\right)\right)^{1 / 2} V_{-n+1 / 2 ; n}\left(\xi_{n}\right) \cdots V_{-1 / 2 ; n}\left(\xi_{n}\right)\right) \\
& \cdot \pi_{e}\left(T^{n, \xi_{n}}(f)\right) \pi_{e}\left(V_{1 / 2 ; n}\left(\xi_{n}\right) \cdots V_{n-1 / 2 ; n}\left(\xi_{n}\right)\right. \\
& \left.\left.\cdot\left(V_{n ; n, \perp}\left(\xi_{n}\right)\right)^{1 / 2}\right)\left(j_{n+1}^{-1} \circ \alpha_{P_{n+1}(n)} \xi_{n}\right)^{\wedge}\right\rangle_{\mathfrak{S}_{e}^{\otimes}}=l_{\xi_{n}}\left(T^{n, \xi_{n}} f\right)
\end{aligned}
$$

for all $f \in C_{\Lambda_{n}}$ and $l_{\Lambda_{n}}$ is the linear functional on $\mathfrak{A}_{0}$ given by

$$
\begin{aligned}
l_{\xi_{n}}(\cdot)= & \frac{1}{Z_{\left(\Lambda_{n}\right), \xi_{n}}^{\Phi}}\left\langle\left(j_{-(n+1)}^{-1}{ }^{\circ} \alpha_{P_{-(n+1)}(n)} \xi_{n}\right)^{\wedge},\right. \\
& \cdot \pi_{e}\left(\left(V_{-n ; n, \perp}\left(\xi_{n}\right)\right)^{1 / 2} V_{-n+1 / 2 ; n}\left(\xi_{n}\right) \cdots V_{-1 / 2 ; n}\left(\xi_{n}\right)\right) \\
& \cdot \pi_{e}(\cdot) \pi_{e}\left(V_{1 / 2 ; n}\left(\xi_{n}\right) \cdots V_{n-1 / 2 ; n}\left(\xi_{n}\right)\left(V_{n ; n, \perp}\left(\xi_{n}\right)\right)^{1 / 2}\right) \\
& \left.\cdot\left(j_{n+1}^{-1} \circ \alpha_{P_{n+1}(n)} \xi_{n}\right)^{\wedge}\right\rangle_{\mathfrak{H}_{e}^{\otimes} .}
\end{aligned}
$$

Next define the linear map $T: C_{0}(\Omega) \rightarrow \mathfrak{U}_{0}$ as the map which maps $f \in C_{0}(\Omega)$ onto $T(f) \in \mathfrak{A}_{0}$, where

$$
T(f)=\lim _{n \rightarrow \infty} T^{n, \xi_{n}}(f)
$$

This limit exists trivially for each $f \in C_{0}(\Omega)$ : for some $n_{0} \in \mathbb{N}$ the sequence $\left\{T^{n, \xi_{n}}(f)\right\}_{\substack{n \geqq n_{0} \\ n \in \mathbb{N}}}$ is a constant sequence with elements which moreover do not depend on the sequence $\left\{\xi_{n}\right\}_{\substack{n \geqq n_{0} \\ n \in \mathbb{N}}}$ of configurations with $\xi_{n} \in \Omega_{\Lambda_{n}^{c}}$.

Let $\mu$ be a Gibbs state for the potential $\Phi$. If $f \in C_{\Lambda_{n}}$ and $m \in \mathbb{N}$ is taken large enough that $T^{m: \xi_{m}}(f)=T(f)$ for each $\xi_{m} \in \Omega_{\Lambda_{m}^{c}}$, then

$$
\begin{aligned}
\mu(f) & =\int_{\Omega_{\Lambda_{m}^{c}}}\left(\alpha_{\Lambda_{m}^{c}} \mu\right)\left(d \xi_{m}\right)\left(\mathbb{E}_{\Lambda_{m}^{c}}^{\Phi} f\right)\left(\xi_{m}\right)=\int_{\Omega_{\Lambda_{m}^{c}}}\left(\alpha_{\Lambda_{m}^{c}} \mu\right)\left(d \xi_{m}\right) l_{\xi_{m}}\left(T^{m, \xi_{m}}(f)\right) \\
& =\int_{\Omega_{\Lambda_{m}^{c}}^{c}}\left(\alpha_{\Lambda_{m}^{c}} \mu\right)\left(d \xi_{m}\right) l_{\xi_{m}}(T(f)) .
\end{aligned}
$$

This shows that one can define a linear functional $l_{\mu}$ on the range of $T$ by setting

for $f \in C_{0}(\Omega)$.

$$
l_{\mu}(T(f))=\lim _{m \rightarrow \infty} \int_{\Omega_{\Lambda_{m}^{c}}}\left(\alpha_{\Lambda_{m}^{c}} \mu\right)\left(d \xi_{m}\right) l_{\xi_{m}}(T(f))
$$

If $S: X \rightarrow Y$ is a map from a set $X$ into a set $Y$, then for $X_{1} \subset X$ we denote by $S X_{1}$ the image of $X_{1}$ under $S$. Furthermore if $A_{1}$ and $A_{2}$ are subsets of an algebra then $A_{1} A_{2}=\left\{a \mid a=a_{1} a_{2}\right.$ with $a_{1} \in A_{1}$ and $\left.a_{2} \in A_{2}\right\}$.

\section{Proposition 1.}

$$
T\left(C_{P_{0} \cup P_{1}} \cap C_{0}(\Omega)\right)=\mathfrak{U}_{0} .
$$

Proof. Take $n \in \mathbb{N}$. It will be shown that

$$
\mathfrak{U}_{P(n)} \subset \text { linear span of } \mathfrak{U}_{P(n+1)}^{\mathrm{diag}} \alpha^{(1)}\left(\mathfrak{U}_{P(n)}^{\mathrm{diag}}\right) .
$$

Then the proposition easily follows. Indeed, note that

$$
\text { linear span of } \mathfrak{U}_{P(n+1)}^{\mathrm{diag}} \alpha^{(1)}\left(\mathfrak{U}_{P(n)}^{\mathrm{diag}}\right)=T\left(C_{P_{0}(n+1) \cup P_{1}(n)}\right) \text {. }
$$


Hence, using (5),

$$
\begin{gathered}
\mathfrak{U}_{0}=\bigcup_{n \in \mathbb{N}} \mathfrak{A}_{P(n)} \subset \bigcup_{n \in \mathbb{N}}\left\{\text { linear span of } \mathfrak{I}_{P(n+1)}^{\mathrm{diag}} \alpha^{(1)}\left(\mathfrak{P}_{P(n)}^{\mathrm{diag}}\right)\right\} \\
=\bigcup_{n \in \mathbb{N}} T\left(C_{P_{0}(n+1) \cup P_{1}(n)}\right)=T\left(C_{P_{0} \cup P_{1}} \cap C_{0}(\Omega) .\right.
\end{gathered}
$$

In order to prove (5) it is useful to consider $\mathfrak{U}_{P(n)}$ and $\mathfrak{U}_{P(n+1)}$ as the matrix algebras by which they were introduced. The linear span of $\mathfrak{U}_{P(n)}^{\text {diag }} V_{1 / 2 ; n, 0} \mathfrak{A}_{P(n)}^{\text {diag }}$ equals $\mathfrak{A}_{P(n)}$, because $\mathfrak{U}_{P(n)}^{\text {diag }}$ is the full algebra of diagonal matrices in $\mathfrak{U}_{P(n)}$ and $V_{1 / 2, n, 0}$ is a matrix that has strictly positive matrix elements. Therefore,

$$
\text { linear span of } \mathfrak{U}_{P(n)}^{\text {diag }} V_{1 / 2 ; n, 0} \mathfrak{U}_{P(n)}^{\text {diag }}\left(V_{1 / 2, n, 0}\right)^{-1}=\mathfrak{U}_{P(n)} \text {. }
$$

On the other hand,

$$
\alpha^{(1)}\left(\mathfrak{U}_{P(n)}^{\mathrm{diag}}\right)=\left(V_{0 ; n+1, \perp}\right)^{1 / 2} V_{1 / 2, n, 0} \mathfrak{\mathcal { H }}_{P(n)}^{\mathrm{diag}}\left(V_{1 / 2, n, 0}\right)^{-1}\left(V_{0 ; n+1, \perp}\right)^{-1 / 2} .
$$

Thus,

$$
\mathfrak{U}_{P(n+1)}^{\text {diag }} \alpha^{(1)}\left(\mathfrak{U}_{P(n)}^{\mathrm{diag}}\right)=\mathfrak{U}_{P(n+1)}^{\mathrm{diag}} V_{1 / 2 ; n, 0} \mathfrak{\mathcal { H }}_{P(n)}^{\mathrm{diag}}\left(V_{1 / 2 ; n, 0}\right)^{-1}\left(V_{0 ; n+1, \perp}\right)^{-1 / 2} .
$$

Hence, by furthermore noting that

$$
\left(V_{0 ; n+1, \perp}\right)^{-1 / 2} \in \text { linear span of } \mathfrak{U}_{P(n+1) \backslash P(n)} \mathfrak{A}_{P(n)}
$$

we obtain,

$$
\begin{aligned}
& \text { linear span of } \mathfrak{Q}_{P(n+1)}^{\mathrm{diag}} \alpha^{(1)}\left(\mathfrak{I}_{P(n)}^{\mathrm{diag}}\right) \\
& =\text { linear span of } \mathfrak{U}_{P(n+1) \backslash P(n)}^{\mathrm{diag}} \mathfrak{A}_{P(n)}\left(V_{0 ; n+1, \perp}\right)^{-1 / 2} \\
& =\text { linear span of } \mathfrak{U}_{P(n+1) \backslash P(n)}^{\mathrm{diag}} \mathfrak{A}_{P(n)} \supset \mathfrak{U}_{P(n)} \cdot \quad \square
\end{aligned}
$$

As a corollary of Proposition 1 one obtains that for each-Gibbs state $\mu$ there exists a unique linear functional $l_{\mu}$ on $\mathfrak{U}_{0}$ such that

$$
l_{\mu} \circ T=\mu \uparrow_{C_{0}(\Omega)} .
$$

Such a pair $\left(\mu, l_{\mu}\right)$ with $\mu$ a state on $C(\Omega)$ and $l_{\mu}$ a linear functional on $\mathfrak{U}_{0}$ which are related as in (6) will be called a canonical pair (for $T$ ). Note that $T \uparrow_{C_{p_{0}} \cap C_{0}(\Omega)}$ is a *-isomorphism onto $\mathfrak{U}_{0}^{\text {diag }}$. Thus the plane $P_{0}$ plays a special role. However one could also consider a formalism in which this role is played by the plane $P_{k}$. In such a formalism one would, instead of considering the functional $l_{\mu}$ associated with a Gibbs state $\mu$ by $T$, consider the functional $l_{\mu}{ }^{\circ} \alpha^{(k)}$ associated with $\mu$ by $\alpha^{(k)^{-1}} \circ T$.

Part b. of the next theorem is for $k=0$ an immediate consequence of Proposition 1 , but in view of the remarks just made it holds for each $k \in \mathbb{Z}$.

\section{Theorem 1.}

a. If $\mu$ is a Gibbs state then there exists a unique linear functional $l_{\mu}$ on $\mathfrak{U}_{0}$ such that

$$
\mu \uparrow_{C_{0}(\Omega)}=l_{\mu} \circ T \text {. }
$$

b. For each $k \in \mathbb{Z}$ a Gibbs state is determined by its restriction to $C_{P_{k} \cup P_{k+1}}$. 
Remark. Proposition 1 implies that for each $g \in C_{0}(\Omega)$ there exists an $f \in C_{P_{0} \cup P_{1}} \cap$ $C_{0}(\Omega)$ such that $T(f-g)=0$. Apart from $g$ the function $f$ only depends on $\Phi$. Hence for each Gibbs state $\mu$,

$$
\mu(f)=\mu(g)
$$

An explicit formula for $f$ given $g$ can be obtained by applying Eq. (8) in the next section.

\section{Decompositions}

In order to exploit the relation $\mu \uparrow_{C_{0}(\Omega)}=l_{\mu} \circ T$ between a Gibbs state $\mu$ and a linear functional $l_{\mu}$ on $\mathfrak{U}_{0}$ further, it is useful to assume that $\Phi$ and $\mu$ have some invariance. Let $\theta_{k}$ be the reflection of $\mathbb{Z}^{d}$ in the plane $\left\{x \in \mathbb{R}^{d} \mid x_{0}=k\right\}$ where $k \in \mathbb{Z}$. Furthermore let $\tau^{(a)}$ be the action of translating $\mathbb{Z}^{d}$ over a $a \in \mathbb{Z}^{d}$. These actions, $\tau^{(a)}$ and $\theta_{k}$, act in a natural way on the spaces $\Omega, C(\Omega), E_{C(\Omega)}$, and on potentials. These actions are also denoted by $\tau^{(a)}$ and $\theta_{k}$. If $G \subset \mathbb{Z}^{d}$ is a subgroup of $\mathbb{Z}^{d}$, then $E_{C(\Omega)}^{G}$ denotes the set of $G$-invariant states on $C(\Omega)$. Furthermore, if $a \in \mathbb{Z}^{d-1}$ then the automorphism of $\mathfrak{A}$ which gives translation by $a \in \mathbb{Z}^{d-1}$ is also denoted by $\tau^{(a)}$. If $H \subset \mathbb{Z}^{d-1}$ is a subgroup then $E_{\mathfrak{N}}^{H}$ denotes the set of $H$-invariant states on $\mathfrak{A}$. In the following $\mathbb{Z}^{d-1}$ is identified with $\left\{x \in \mathbb{Z}^{d} \mid x_{0}=0\right\}$ by the correspondence

$$
\mathbb{Z}^{d-1} \ni y=\left(y_{1}, \ldots, y_{d-1}\right) \leftrightarrow\left(0, y_{1}, \ldots, y_{d-1}\right) \in \mathbb{Z}^{d} .
$$

In particular subgroups of $\mathbb{Z}^{d-1}$ are in this way viewed as subgroups of $\mathbb{Z}^{d}$. Henceforth we assume the potential $\Phi$ meets Condition $C_{2}$.

Condition $C_{2}$. The potential $\Phi$ is reflectionally invariant with respect to reflections in the planes $\left\{x \in \mathbb{Z}^{d} \mid x_{j}=k\right\} \quad k \in \mathbb{Z}$, and the potential $\Phi$ has an invertible contact operator in each coordinate direction of $\mathbb{Z}^{d}$.

Note that Condition $C_{1}$ expresses that $\Phi$ has invertible contact matrices in the 0 -direction. Condition $C_{2}$ therefore includes Condition $C_{1}$. Also note that $\theta_{0} \circ \theta_{1}=\tau_{0}^{2}$, where $\tau_{0}=\tau^{((1,0, \ldots, 0))}$ with $(1,0, \ldots, 0) \in \mathbb{Z}^{d}$. Thus Condition $C_{2}$ implies that $\Phi$ is $\mathbb{Z}_{e v}^{d}$-invariant, where $\mathbb{Z}_{e v}$ is the set of even integers. The $\mathbb{Z}_{e v}^{d}$-invariance of $\Phi$ implies

$$
v_{k+2+1 / 2,0}(y)=v_{k+1 / 2,0}(y) \text { for all } k \in \mathbb{Z}, \quad y \in \mathbb{Z}^{d-1}
$$

and

$$
v_{k+1 / 2,0}(y)=v_{k+1 / 2,0}\left(y+y^{\prime}\right) \quad \text { for all } k \in \mathbb{Z}, \quad y \in \mathbb{Z}^{d-1} \text { and } y^{\prime} \in \mathbb{Z}_{e v}^{d-1} .
$$

Reflectional invariance implies furthermore

$$
v_{1 / 2,0}(y)^{*}=v_{-1 / 2,0}(y) \text { for all } y \in \mathbb{Z}^{d-1} .
$$

Similarly for all $k \in \mathbb{Z}$ and $[y, z] \in \bigcup_{n \in \mathbb{N}} \widetilde{P}_{n}$,

$$
v_{k+2, \perp}([y, z])=v_{k, \perp}([y, z])
$$


and

$$
\tau^{(a)}\left(v_{k, \perp}([y, z])\right)=v_{k, \perp}([y+a, z+a])
$$

for all $a \in \mathbb{Z}_{e v}^{d-1}$. Also for all $k \in \mathbb{Z}, y \in \mathbb{Z}^{d-1}$ and $a \in \mathbb{Z}_{e v}^{d-1}$

$$
\tau^{(a)}\left(w_{k+2, \perp}(y)\right)=w_{k, \perp}(y+a) .
$$

Furthermore $v_{k, \perp}([y, z])^{*}=v_{k, \perp}([y, z])$ and $w_{k}(y)^{*}=w_{k}(y)$. The following lemma states some properties of the map $T: C_{0}(\Omega) \rightarrow \mathfrak{A}_{0}$ which are easy consequences of the above stated properties of the operators which constitute the transfer matrix.

Lemma 1. Take $f \in C_{0}(\Omega)$. The map $T: C_{0}(\Omega) \rightarrow \mathfrak{U}_{0}$ has the following properties.

1. $T\left(\overline{\left(\theta_{0} f\right)}\right)=T(f)^{*}$

2. $T\left(\tau_{0}^{2} f\right)=\alpha^{(2)}(T(f))$,

3. $T\left(\tau_{j}^{2} f\right)=\tau_{j}^{2}(T(f))$ for $j=1, \ldots, d-1$, where $\tau_{j}=\tau^{\left(a^{(j)}\right)}$ with $a^{(j)} \in \mathbb{Z}^{d-1}$ given by components $a_{i}^{(j)}=\delta_{i, j} ; i=0, \ldots, d-1$.

Proof. Define $\alpha_{\delta}: \mathfrak{U}_{0} \rightarrow \mathfrak{U}_{0}$ for $\delta= \pm 1$ as the map which maps $\sigma \in \mathfrak{U}_{0}$ onto $\alpha_{\delta}(\sigma) \in \mathfrak{U}_{0}$ given by

$$
\alpha_{\delta}(\sigma)=\lim _{n \rightarrow \infty} V_{\delta 1 / 2, n} \sigma\left(V_{\delta 1 / 2 ; n}\right)^{-1}
$$

Then, if $1 \leqq k \in \mathbb{N}$,

$$
\begin{aligned}
\alpha^{(k)} & =\alpha_{+1} \circ \alpha_{-1} \circ \cdots \circ \alpha_{-(-1)^{k}}, \\
\alpha^{(-k)} & =\alpha_{-1}^{-1} \circ \alpha_{+1}^{-1} \circ \cdots \circ \alpha_{(-1)^{k}}^{-1} .
\end{aligned}
$$

Since $\left(V_{1 / 2, n}\right)^{*}=V_{-1 / 2, n}$, one obtains

$$
\left(\alpha_{+1}(\sigma)\right)^{*}=\alpha_{-1}^{-1}\left(\sigma^{*}\right) \text { for } \sigma \in \mathfrak{P}_{0}
$$

Thus $\left(\alpha^{(k)}(\sigma)\right)^{*}=\alpha^{(-k)}\left(\sigma^{*}\right)$. Using the definition of $T$ the assertions of the lemma follow.

Condition $C_{2}$ is sufficient to invoke the following theorem which can be found in $[11]$.

Theorem 2. Let $\mu \in \mathscr{G}_{\Phi}$ be invariant under a subgroup of $\mathbb{Z}^{d}$ with finite index in $\mathbb{Z}^{d}$ and assume that $\mu$ is $\theta_{k}$-invariant for each $k \in \mathbb{Z}$. Let $\bar{\mu}$ be the unique state on $C(\Omega)$ that satisfies:

1. $\bar{\mu}$ is $\theta_{k}$-invariant for each $k \in \mathbb{Z}$;

2. $\bar{\mu}$ has the Markov property with respect to $P_{k}$ for each $k \in \mathbb{Z}$;

3. $\bar{\mu} \uparrow_{C_{P_{0} \cup P_{1}}}=\mu \uparrow_{C_{P_{0} \cup P_{1}}}$.

Then $\bar{\mu}$ is a Gibbs state.

The following definition of the Markov property with respect to $P_{k}$ is used. If $\mu$ is a state on $\mathfrak{B}$, let $\mathbb{E}_{\Lambda}^{\mu}$ be the orthogonal projection on $L^{2}(\mu ; \mathfrak{B})$ with range $L^{2}\left(\mu ; \mathfrak{B}_{\Lambda}\right)$, where $\Lambda \subset \mathbb{Z}^{d}$. The state $\mu$ is said to have the Markov property with 
respect to $P_{k}$ if

$$
\mathbb{E}_{P_{k}}^{\mu} f=\mathbb{E}_{\left(\mathbb{Z}^{d}\right)_{k,-}}^{\mu} f
$$

for all $f \in L^{2}\left(\mu ; \mathfrak{B}_{\left(\mathbb{Z}^{d}\right)_{k,+}}\right)$, where $\left(\mathbb{Z}^{d}\right)_{k, \delta}=\left\{x \in \mathbb{Z}^{d} \mid \delta\left(x_{0}-k\right) \geqq 0\right\}$ and $\delta= \pm$.

Now Theorem 1 says that each Gibbs state is determined by its restriction to $C_{P_{0} \cup P_{1}}$. Hence the states $\mu$ and $\bar{\mu}$ which appear in Theorem 2 are equal. Note that if $\mu$ is a state which satisfies $\mu=\mu^{\circ} \tau_{0}^{2}$ and $\mu=\mu^{\circ} \theta_{0}$, then $\mu=\mu \circ \theta_{k}$ for all $k \in \mathbb{Z}$ because $\theta_{k}=\tau_{0}^{2 k} \circ \theta_{0}$. Therefore one obtains from Theorem 1 and Theorem 2 the next corollary.

Corollary 1. Choose $l \in \mathbb{Z}$. Let $\mu \in \mathscr{G}_{\Phi} \cap E_{C(\Omega)}^{\mathbb{Z}_{\mathrm{ev}}^{d}}$ be $\theta_{i}$-invariant. Then $\mu$ has the Markov property with respect to $P_{k}$ for all $k \in \mathbb{Z}$.

Lemma 2. Choose $k \in \mathbb{Z}$. Let $\mu$ be a state on $C(\Omega)$ which is $\theta_{k}$-invariant and which has the Markov property with respect to $P_{k}$. Then $\mu$ is $\theta_{k}$-reflection positive, i.e.,

$$
\mu\left(\overline{\left(\theta_{k} f\right)} f\right) \geqq 0 \quad \text { for all } f \in C_{\left(\mathbb{Z}^{d}\right)_{k+}} .
$$

Proof. See [8], pp. 104/105.

Let $\left(\mu, l_{\mu}\right)$ be a canonical pair. Then for $f \in C_{\left(\mathbb{Z}^{d}\right)_{0,+}} \cap C_{0}(\Omega)$,

$$
\mu\left(\overline{\left(\theta_{0} f\right)} f\right)=l_{\mu}\left(T(f)^{*} T(f)\right) .
$$

Thus, using Proposition 1, one sees that the following assertions are equivalent:

1. $\mu$ is $\theta_{0}$-reflection positive;

2. $l_{\mu}$ is a state on $\mathfrak{U}_{0}$.

In case $l_{\mu}$ is a state on $\mathfrak{A}_{0}$ then $l_{\mu}$ has a unique extension to a state on $\mathfrak{U}$. When a state $\mu$ on $C(\Omega)$ and a state $\omega$ on $\mathfrak{A}$ satisfy

$$
\mu \Upsilon_{C_{0}(\Omega)}=\omega \circ T
$$

on $_{7^{d}} C_{0}(\Omega)$, the pair $(\mu, \omega)$ will be called a canonical state pair (for $T$ ). If $\mu \in \mathscr{G}_{\Phi} \cap$ $E_{C(\Omega)}^{\mathbb{Z}_{\mathrm{ev}}^{d}}$ is $\theta_{0}$-invariant, then by Corollary 1 and Lemma 2 the state $\mu$ is part of a canonical state pair. In particular this shows that the set of canonical state pairs is nonempty.

If $\omega$ is a state on $\mathfrak{A}$ we denote by $\left(\mathfrak{H}_{\omega}, \pi_{\omega}, \Omega_{\omega}\right)$ a G.N.S. triple for $\omega$, that is, $\mathfrak{H}_{\omega}$ is a Hilbert space, $\pi_{\omega}$ is a *-representation of $\mathfrak{U}$ as bounded operators on $\mathfrak{H}_{\omega}, \Omega_{\omega}$ is a unit vector in $\mathfrak{H}_{\omega}$ which is cyclic for $\pi_{\omega}(\mathfrak{U})$ and the triple is such that

$$
\omega(\sigma)=\left\langle\Omega_{\omega}, \pi_{\omega}(\sigma) \Omega_{\omega}\right\rangle
$$

for $\sigma \in \mathfrak{U}$ and $\langle\cdot, \cdot\rangle$ is the inner product on $\mathfrak{H}_{\omega} \times \mathfrak{H}_{\omega}$. Define $\mathfrak{M}_{\omega}=\left(\pi_{\omega}(\mathfrak{A})\right)^{\prime \prime}$, the center $\mathfrak{Z}_{\omega}$ of $\mathfrak{M}_{\omega}: \mathfrak{Z}_{\omega}=\mathfrak{M}_{\omega} \cap \mathfrak{M}_{\omega}^{\prime}$, and $\mathfrak{M}_{\omega}^{\text {diag }}=\left(\pi_{\omega}\left(\mathfrak{2} \mathfrak{I}^{\text {diag }}\right)\right)^{\prime \prime}$.

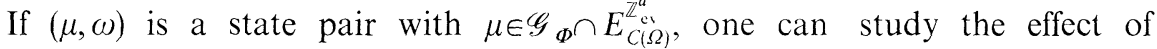
decomposing $\omega$ into other states on the possibilities of decomposing $\mu$. This will be done in the subsequent part of this paper, but first we remark that the state $\omega$ on $\mathfrak{U}_{0}$ can be introduced slightly differently from the way this state was introduced above starting from a $\theta_{0}$-reflection positive Gibbs state $\mu$. Let $(\mu, \omega)$ be a canonical state pair with $\mu \in \mathscr{G}_{\Phi}$. The Hilbert space $\mathfrak{H}_{\omega}$ is canonically isomorphic to the Hilbert 
space $\tilde{\mathfrak{H}}$ which is constructed from the positive sesquilinear function $b_{0}(\cdot, \cdot)$ on $L^{2}\left(\mu ; \mathfrak{B}_{\left(\mathbb{Z}^{d}\right)_{0,+}}\right) \times L^{2}\left(\mu ; \mathfrak{B}_{\left(\mathbb{Z}^{d}\right)_{0,+}}\right)$ given by

$$
b_{0}(f, g)=\left\langle\theta_{0} f, g\right\rangle_{\mu},
$$

where $f, g \in L^{2}\left(\mu ; \mathfrak{B}_{\left.\left(\mathbb{Z}^{d}\right)_{0_{+}}\right)}\right.$and $\langle\cdot, \cdot\rangle_{\mu}$ denotes the inner product on $L^{2}\left(\mu ; \mathfrak{B}_{\mathbb{Z}^{d}}\right) \times$ $L^{2}\left(\mu ; \mathfrak{B}_{\mathbb{Z}^{d}}\right)$. See Fredenhagen's paper [3]. In [3] the construction of the state $\omega$ from the $\theta_{0}$-reflection positive Gibbs state $\mu$ uses the inverse $E_{+}$of $T \uparrow_{C_{0}(\Omega) \cap C_{P_{0} \cup P_{1}}}$. Explicitly $E_{+}: \mathfrak{U}_{0} \rightarrow C_{0}(\Omega) \cap C_{P_{0} \cup P_{1}}$, which maps $\sigma \in \mathfrak{U}_{0}$ onto $E_{+}(\sigma) \in C_{0}(\Omega) \cap C_{P_{0} \cup P_{1}}$ is given by

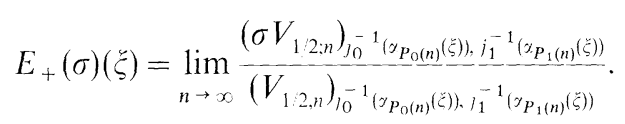

The state $\omega$ is now introduced by

$$
\omega(\sigma)=\mu\left(E_{+}(\sigma)\right)
$$

for $\sigma \in \mathfrak{U}_{0},[3]$.

Consider a canonical state pair $(\mu, \omega)$ where $\mu$ is $\tau^{(a)}$-invariant with $a \in \mathbb{Z}_{e v}^{d-1}$. Since $T\left(\tau^{(a)} f\right)=\tau^{(a)}(T(f))$, see Lemma 1.3 , the state $\omega$ is $\tau^{(a)}$-invariant and there thus exists a unique unitary operator $U_{a}$ on $\mathfrak{H}_{\omega}$ such that

$$
U_{a} \pi_{\omega}(\sigma) \Omega_{\omega}=\pi_{\omega}\left(\tau^{(a)}(\sigma)\right) \Omega_{\omega}
$$

for all $\sigma \in \mathfrak{A}$. Now let $(\mu, \omega)$ be a canonical state pair where $\mu$ is $\tau_{0}^{2 n}$-invariant with $n \in \mathbb{N}$. Then there eixsts a unique operator $P_{\infty}^{(2 n)}$ on $\mathfrak{H}_{\omega}$ which satisfies

$$
P_{\infty}^{(2 n)} \pi_{\omega}(\sigma) \Omega_{\omega}=\pi_{\omega}\left(\alpha^{(2 n)}(\sigma)\right) \Omega_{\omega}
$$

for all $\sigma \in \mathfrak{A}_{0}$. By arguments in [9], see also Schor's paper [10], Eq. (9) defines $P_{\infty}^{(2 n)}$ as a self-adjoint contraction with $\left\|P_{\infty}^{(2 n)}\right\|=1$.

Actually the paper [10] does not consider Eq. (9) precisely. This paper uses the Hilbert space $\hat{\mathfrak{H}}$ and also one considers the case of a reflectionally as well as translationally invariant $\Phi$. In such cases, if $(\mu, \omega)$ is a canonical state pair with $\mu \circ \tau_{0}=\mu$, one can introduce the operator $P_{\infty}$ on $\mathfrak{H}_{\omega}$ by

$$
P_{\infty} \pi_{\omega}(\sigma) \Omega_{\omega}=\pi_{\omega}(\alpha(\sigma)) \Omega_{\omega},
$$

where $\alpha=\alpha_{+1}\left(=\alpha_{-1}\right)$. Here $\alpha_{+1}$ and $\alpha_{-1}$ are the maps on $\mathfrak{U}_{0}$ which were introduced in the proof of Lemma 1 (and $\alpha_{+1}=\alpha_{-1}$ by the assumed translational invariance of $\Phi$ ). It is Eq. (10) to which the arguments in [10], which give that $P_{\infty}$ is a self-adjoint contraction, immediately apply. However the case described by (10) is completely analogous to the one given by (9).

The operator $P_{\infty}$ is called the infinite-volume transfer operator. If $P_{\infty}^{(2 n)}$ is defined by (9), then clearly $P_{\infty}^{(2 n)}=\left(P_{\infty}\right)^{2 n}$. Since the state $\mu$ is not assumed to be translationally invariant the "powers" of the infinite-volume transfer operator, $P_{\infty}^{(2 n)}$, which are defined by (9), are considered in the following.

The operator $P_{\infty}^{(2 n)}$ is positive if and only if $\mu$ is $\theta_{n}$-reflection positive. Moreover $\pi_{\omega}\left(\mathfrak{U}_{0}\right) \Omega_{\omega}$ is in the range of $P_{\infty}^{(2 n)}$. Therefore 0 is not in the point spectrum. Hence $P_{\infty}^{(2 n)}$ has an (unbounded) inverse $P_{\infty}^{(2 n)^{-1}}$, cf. [3]. Let $H \subseteq \mathbb{Z}_{e v}^{d-1}$ be a group and $n \in \mathbb{Z}$. The group $H_{2 n} \subseteq \mathbb{Z}_{e v}^{d}$ is defined as the group generated by $H$ and $(2 n, 0, \ldots, 0) \in \mathbb{Z}^{d}$. 
Write $H_{0}=H$. Furthermore define

$$
R_{\omega}\left(H_{2 n}\right)=\left\{U_{a} \mid a \in H\right\} \cup\left\{P_{\infty}^{(2 n)}\right\},
$$

when the state $\omega$ on $\mathfrak{A}$ is a part of a canonical state pair $(\mu, \omega)$ with a state $\mu$ on $C(\Omega)$ that is $H_{2 n}$-invariant. The von Neumann algebra $\mathfrak{N}_{\omega}\left(H_{2 n}\right)$ is defined as

$$
\mathfrak{N}_{\omega}\left(H_{2 n}\right)=\left(\mathfrak{M}_{\omega} \cup R_{\omega}\left(H_{2 n}\right)\right)^{\prime} .
$$

Lemma 3. Let $(\mu, \omega)$ be a canonical state pair. The following assertions are equivalent:

1. $\mu$ has the Markov property with respect to $P_{0}$,

2. $\mathfrak{M}_{\omega}^{\mathrm{diag}} \Omega_{\omega}$ is dense in $\mathfrak{H}_{\omega}$.

If one of these assertions is true, then

$$
3_{\omega}=\mathfrak{M}_{\omega}^{\prime} .
$$

Proof. The equivalence of the assertions 1. and 2. is well-known. See e.g. remarks made in Chap. IV in [12]. If assertion 2. is true, then $\mathfrak{M}_{\omega}^{\mathrm{diag}}=\left(\mathfrak{M}_{\omega}^{\mathrm{diag}}\right)^{\prime}$ and the last claim of Lemma 3 immediately follows.

In case one of the assertions in Lemma 3 holds, let $I_{0}^{\mu}: L^{2}\left(\mu ; \mathfrak{B}_{P_{0}}\right) \rightarrow \mathfrak{H}_{\omega}$ be the unique unitary operator which maps $f \in C_{P_{0}} \cap C_{0}(\Omega) \subset L^{2}\left(\mu ; \mathfrak{B}_{P_{0}}\right)$ onto $I_{0}^{\mu} f \in \mathfrak{H}_{\omega}$ given by

$$
I_{0}^{\mu} f=\pi_{\omega}(\hat{f}) \Omega_{\omega} .
$$

Henceforth, if one of the assertions in Lemma 3 is true, the Hilbert space $L^{2}\left(\mu ; \mathfrak{B}_{P_{0}}\right)$ is identified with the Hilbert space $\mathfrak{H}_{\omega}$ by means of $I_{0}^{\mu}$. Then $L^{\infty}\left(\mu ; \mathfrak{B}_{P_{0}}\right)=\mathfrak{M}_{\omega}^{\text {diag }}$ when considering $L^{\infty}\left(\mu ; \mathfrak{B}_{P_{0}}\right)$ as the set of bounded multiplication operators on $L^{2}\left(\mu ; \mathfrak{B}_{P_{0}}\right)$. If the operators $U_{a}$ for $a \in \mathbb{Z}_{e v}^{d-1}$ and $P_{\infty}^{(2 n)}$ are defined, the operator $U_{a}$ is equal to $\tau^{(a)}$ under the identification by $I_{0}^{\mu}$ and $P_{\infty}^{(2 n)}$ equals $\mathbb{E}_{P_{0}} \circ \tau_{0}^{(2 n)}$, where the last operator is considered as an operator on $L^{2}\left(\mu ; \mathfrak{B}_{P_{0}}\right)$. Furthermore, by this identification, for $f \in C_{\left(\mathbb{Z}^{d}\right)_{0 .+}} \cap C_{0}(\Omega)$ we have $\pi_{\omega}(T(f)) \Omega_{\omega}=\mathbb{E}_{P_{0}(f)}$.

Let $(\mu, \omega)$ be a canonical state pair. Then for $f \in C_{0}(\Omega)$ one has

$$
\begin{aligned}
\mu\left(\theta_{0} f\right) & =\overline{\mu\left(\overline{\left.\theta_{0} f\right)}\right.}=\left\langle\overline{\Omega_{\omega}, \pi_{\omega}\left(T \overline{\left(\theta_{0} f\right)}\right) \Omega_{\omega}}\right\rangle=\overline{\left\langle\Omega_{\omega}, \pi_{\omega}(T(f))^{*} \Omega_{\omega}\right\rangle} \\
& =\left\langle\Omega_{\omega}, \pi_{\omega}(T(f)) \Omega_{\omega}\right\rangle=\mu(f) .
\end{aligned}
$$

Therefore $\mu$ is $\theta_{0}$-invariant and thus has the Markov property with respect to $P_{0}$. This means that the first assertion of Lemma 3 is true, in particular therefore $\mathfrak{M}_{\omega}^{\prime}=3_{\omega}$.

Lemma 4. Let $H \subset \mathbb{Z}_{\mathrm{ev}}^{d-1}$ be a group and $n \in \mathbb{N}$. Assume $H_{2 n}$ is nontrivial. Let $(\mu, \omega)$ be a canonical state pair with $\mu \in \mathscr{G}_{\Phi} \cap E_{C(\Omega)}^{\mathbb{Z}_{\mathrm{ev}}}$ Then,

a. $\left\{A \Omega_{\omega} \mid A \in \mathfrak{N}_{\omega}\left(H_{2 n}\right)\right\}^{\text {clos }}=\mathfrak{H}_{\omega}^{H_{2 n}}$, where

$$
\mathfrak{H}_{\omega}^{H_{2 n}}=\left\{\psi \in \mathfrak{S}_{\omega} \mid P_{\infty}^{(2 n)} \psi=\psi\right\}
$$

and $U_{a} \psi=\psi$ for all $\left.a \in H\right\}$ and $X^{\text {clos }}$ denotes the closure of a set $X \subset \mathfrak{H}_{\omega}$.

b. $\mathfrak{N}_{\omega}\left(H_{2 n}\right)=\left(\mathfrak{M}_{\omega}^{\mathrm{diag}} \cup R_{\omega}\left(H_{2 n}\right)\right)^{\prime}$. 
Proof. Note that by the remarks made just prior to this lemma the state $\mu$ is $\theta_{0}$-invariant and $\mathfrak{M}_{\omega}^{\prime}=3_{\omega}$.

a. Let $\psi \in \mathfrak{H}_{\omega}^{H_{2 n}}$. Then $\mathbb{E}_{P_{0}}^{\mu} \circ \tau_{0}^{2 n} \psi=\psi \in L^{2}\left(\mu ; \mathfrak{B}_{P_{0}}\right) \subset L^{2}(\mu ; \mathfrak{B})$. But $\mathbb{E}_{P_{0}}^{\mu}$ is an orthogonal projection and $\tau_{0}^{2 n}$ acts isometrically, therefore

$$
\tau_{0}^{2 n} \psi=\psi
$$

Furthermore $\tau^{(a)} \psi=\psi$ for all $a \in H$. Thus $\psi$ is invariant under the action by elements from $H_{2 n}$. By proposition 3.6 in [14] one obtains then

$$
\psi \in \bigcap_{\substack{\Lambda \subset \mathbb{Z}^{d} \\ \Lambda \text { finite }}} L^{2}\left(\mu ; \mathfrak{B}_{\Lambda^{c}}\right)
$$

If $\Lambda \subset \mathbb{Z}^{d}$ is finite, denote by $E_{\Lambda^{c}}$ the bounded operator on $L^{2}(\mu ; \mathfrak{B})$ which maps $f \in L^{2}(\mu, \mathfrak{B})$ onto $E_{\Lambda^{c}} f \in L^{2}(\mu ; \mathfrak{B})$ where $E_{\Lambda^{c}} f$ is given by

$$
\left(E_{\Lambda^{c}} f\right)(\xi)=\sum_{\xi_{\Lambda} \in \Omega_{\Lambda}} f\left(\xi_{\Lambda} \vee \alpha_{\Lambda^{c}}(\xi)\right)
$$

for $\xi \in \Omega$.

The operator $E_{A^{c}}$ is well-defined on $L^{2}(\mu ; \mathfrak{B})$ since $\mu$ is a Gibbs measure. One has $E_{\Lambda^{c}} \psi=\psi$ for all $\Lambda$ finite. Let $\left\{g_{k}\right\}_{k \in \mathbb{N}}$ be a sequence in $C_{0}(\Omega) \cap C_{P_{0}}$ which converges in $L^{2}(\mu ; \mathfrak{B})$ to $\psi$ :

$$
\psi=\lim _{k \rightarrow \infty} g_{k}
$$

If $\Lambda$ is finite,

$$
E_{\Lambda^{c}} g_{k} \in C_{0}(\Omega) \cap C_{P_{0} \cap \Lambda^{c}}
$$

and

$$
\psi=\lim _{k \rightarrow \infty} E_{\Lambda^{c}} g_{k} .
$$

Hence $\psi \in L^{2}\left(\mu ; \mathfrak{B}_{P_{0} \cap \Lambda^{c}}\right)$. Therefore

$$
\psi \in \bigcap_{\substack{\Lambda \text { finite } \\ \Lambda \subset \mathbb{Z}^{d}}} L^{2}\left(\mu ; \mathfrak{B}_{P_{0} \cap \Lambda^{c}}\right)
$$

If $m \in \mathbb{N}$ define $\psi_{m} \in L^{\infty}(\mu ; \mathfrak{B})$ by

$$
\psi_{m}(\xi)=\left\{\begin{array}{ll}
\psi(\xi) & \text { if }|\psi(\xi)| \leqq m \\
0 & \text { otherwise }
\end{array} .\right.
$$

Then $\psi_{m} \in \bigcap_{\Lambda \text { finte }} L^{\infty}\left(\mu ; \mathfrak{B}_{P_{0} \cap \Lambda^{c}}\right)$ and moreover $\tau^{(a)} \psi_{m}=\psi_{m}$ for $a \in H$ and $\tau_{0}^{2 n} \psi_{m}=\psi_{m}$. One readily see that $\psi_{m} \in\left\{A \Omega \mid A \in \mathbb{Z}^{d} \mathfrak{N}_{\omega}\left(H_{2 n}\right)\right\}$, and since $\psi_{m}$ converges to $\psi$ in $L^{2}(\mu ; \mathfrak{B})$ one concludes that

$$
\psi \in\left\{A \Omega_{\omega} \mid A \in \mathfrak{N}_{\omega}\left(H_{2 n}\right)\right\}^{\text {clos }} .
$$

Since the inclusion

$$
\left\{A \Omega_{\omega} \mid A \in \mathfrak{N}_{\omega}\left(H_{2 n}\right)\right\}^{\text {clos }} \subset \mathfrak{H}_{\omega}^{H_{2 n}}
$$

is obvious, part a. of Lemma 4 has been proven. 
b. Take $A \in\left(\mathfrak{M}_{\omega}^{\mathrm{d} a a g} \cup R_{\omega}\left(H_{2 n}\right)\right)^{\prime}$. Then $A \in \mathfrak{M}_{\omega}^{\mathrm{diag}}$, since $\left(\mathfrak{M}_{\omega}^{\text {diag }}\right)^{\prime}=\mathfrak{M}_{\omega}^{\text {diag }}$. Furthermore $U_{a} A \Omega_{\omega}=A \Omega_{\omega}$ for all $a \in H$ and $P_{\infty}^{(2 n)} A \Omega_{\omega}=A \Omega_{\omega}$. By arguments like the ones used in the proof of part a. of this lemma it follows that $A \in 3_{\omega}$. (Note that $\left.\mathfrak{Z}_{\omega}=\bigcap_{\substack{\Lambda \subset \mathbb{Z}^{d} \\ \Lambda \text { finite }}} L^{\infty}\left(\mu ; \mathfrak{B}_{\Lambda^{c} \cap P_{0}}\right).\right)$ Therefore $A \in \mathfrak{N}_{\omega}\left(H_{2 n}\right)$. The inclusion

$$
\mathfrak{N}_{\omega}\left(H_{2 n}\right) \subset\left(\mathfrak{M}_{\omega}^{\mathrm{diag}} \cup R_{\omega}\left(H_{2 n}\right)\right)^{\prime}
$$

being obvious, part b. of Lemma 4 has been proven.

Lemma 5. Let $(\mu, \omega)$ be a canonical state pair where $\mu$ has the Markov property with respect to $P_{0}$. Let $H \subseteq \mathbb{Z}_{\mathrm{ev}}^{d-1}$ be a nontrivial subgroup and $n \in \mathbb{N}$. Assume $\mu$ is $H_{2 n}$-invariant and $P_{\infty}^{(2 n)} \geqq 0$. Then $P_{\infty}^{(2 n)} \in\left(\mathfrak{N}_{\omega}(H)\right)^{\prime}$.

Proof. Take $A \in \mathfrak{N}_{\omega}(H) \subset 3_{\omega}$. Then there exists a unique $B \in \mathfrak{M}_{\omega}^{\text {diag }}$ such that $P_{\infty}^{(2 n)}$ $A \Omega_{\omega}=B \Omega_{\omega}$. This is most easily seen by viewing $P_{\infty}^{(2 n)}$ as $\mathbb{E}_{P_{0}}^{\mu}{ }^{\circ} \tau_{1}^{2 n}$ and noting that $\mathbb{E}_{P_{0}}^{\mu}$ maps $L^{\infty}$ functions onto $L^{\infty}$ functions (it is a conditional expectation). The uniqueness is a consequence of $\Omega_{\omega}$ being separating for $\mathfrak{M}_{\omega}^{\text {diag }}$. Moreover one easily sees $P_{\infty}^{(2 n)} A^{*} \Omega_{\omega}=B^{*} \Omega_{\omega}$. But $U_{a} P_{\infty}^{(2 n)}=P_{\infty}^{(2 n)} U_{a}$ for all $a \in H$. Hence

$$
U_{a} B \Omega=B \Omega \text {. }
$$

Therefore $B \in \mathfrak{N}_{\omega}(H) \subset 3_{\omega}$, see (the proof of) Lemma 4. Thus, if $\sigma \in \mathfrak{U}_{0}$,

$$
\begin{aligned}
& \left\langle A \Omega_{\omega}, P_{\infty}^{(2 n)^{-1}} \pi_{\omega}(\sigma) \Omega_{\omega}\right\rangle=\left\langle A \Omega_{\omega}, \pi_{\omega}\left(\alpha^{(2 n)^{-1}}(\sigma)\right) \Omega_{\omega}\right\rangle \\
& \quad=\left\langle\pi_{\omega}\left(\alpha^{(2 n)}\left(\sigma^{*}\right)\right) \Omega_{\omega^{\prime}} A^{*} \Omega_{\omega}\right\rangle=\left\langle P_{\infty}^{(2 n)} \pi_{\omega}\left(\sigma^{*}\right) \Omega_{\omega^{\prime}} A^{*} \Omega_{\omega}\right\rangle \\
& \quad=\left\langle\pi_{\omega}\left(\sigma^{*}\right) \Omega_{\omega^{\prime}} B^{*} \Omega_{\omega}\right\rangle=\left\langle B \Omega_{\omega^{\prime}} \pi_{\omega}(\sigma) \Omega_{\omega}\right\rangle=\left\langle P_{\infty}^{(2 n)} A \Omega_{\omega^{\prime}} \pi_{\omega}(\sigma) \Omega_{\omega}\right\rangle .
\end{aligned}
$$

Since $\pi_{\omega}\left(\mathfrak{U}_{0}\right) \Omega_{\omega}$ is a core for $P_{\infty}^{(2 n)^{-1}}$, this implies that $A \Omega_{\omega}$ is in the domain of $P_{\infty}^{(2 n)^{-1}}$. Moreover $P_{\infty}^{(2 n)^{-1}} A \Omega_{\omega}=P_{\infty}^{(2 n)} A \Omega_{\omega}$, that is, $P_{\infty}^{(2 n)^{2}} A \Omega_{\omega}=A \Omega_{\omega}$. Since $P_{\infty}^{(2 n)}$ is positive this implies $P_{\infty}^{(2 n)} A \Omega_{\omega}=A \Omega_{\omega}$. But then for all $\sigma, \sigma^{\prime} \in \mathfrak{U}_{0}$,

$$
\begin{aligned}
& \left\langle\pi_{\omega}(\sigma) \Omega_{\omega^{\prime}} P_{\infty}^{(2 n)} A^{*} \pi_{\omega}\left(\sigma^{\prime}\right) \Omega_{\omega}\right\rangle=\left\langle\pi_{\omega}\left(\alpha^{(2 n)}(\sigma)\right) \Omega_{\omega^{\prime}} A^{*} \pi_{\omega}\left(\sigma^{\prime}\right) \Omega_{\omega}\right\rangle \\
& \left.\quad=\left\langle A \Omega_{\omega^{\prime}} \pi_{\omega}\left(\left(\alpha^{(2 n)^{-1}}\left(\sigma^{*}\right)\right) \sigma^{\prime}\right) \Omega_{\omega}\right\rangle=\left\langle A \Omega_{\omega^{\prime}} P_{\infty}^{(2 n)} \pi_{\omega}\left(\left(\alpha^{(2 n)}\right)^{-1}\left(\sigma^{*}\right)\right) \sigma^{\prime}\right) \Omega_{\omega}\right\rangle \\
& \quad=\left\langle A \Omega_{\omega^{\prime}} \pi_{\omega}\left(\sigma^{*} \alpha^{(2 n)}\left(\sigma^{\prime}\right)\right) \Omega_{\omega}\right\rangle=\left\langle\pi_{\omega}(\sigma) \Omega_{\omega^{\prime}} A^{*} P_{\infty}^{(2 n)} \pi_{\omega}\left(\sigma^{\prime}\right) \Omega_{\omega}\right\rangle .
\end{aligned}
$$

Hence $P_{\infty}^{(2 n)} A^{*}=A^{*} P_{\infty}^{(2 n)}$. It follows that

$$
P_{\infty}^{(2 n)} \in\left(\mathfrak{M}_{\omega}(H)\right)^{\prime}
$$

Let $(\mu, \omega)$ be a canonical state pair where $\mu$ has the Markov property with respect to $P_{0}$. For $Q \in 3_{\omega}$ define $\omega_{Q}$ as the linear functional on $\mathfrak{A}$ given by

$$
\omega_{Q}(\sigma)=\left\langle\Omega_{\omega}, \pi_{\omega}(\sigma) Q \Omega_{\omega}\right\rangle
$$

for $\sigma \in \mathfrak{A}$. Furthermore define $\mu_{Q}$ as the linear functional on $C(\Omega)$ given by

$$
\mu_{Q}(f)=\int \mathrm{d} \mu Q \cdot f
$$

for $f \in C(\Omega)$ : Then

$$
\mu_{Q} \Upsilon_{C_{0}(\Omega)}=\omega_{Q} \circ T
$$


For results and definitions from (noncommutative) decomposition theory which are used below the reader is referred to Chap. IV in the book [13] and the references to the research papers given there.

Lemma 6. Let $(\mu, \omega)$ be a canonical state pair, where $\mu$ has the Markov property with respect to $P_{Q}$. Let $\mathfrak{R}$ be a von Neumann subalgebra of $\mathcal{Z}_{\omega}=\mathfrak{M}_{\omega}^{\prime}$. Let $v_{\mathfrak{R}}$ be the orthogonal measure on $E_{\mathfrak{Q}}$ which corresponds to $\mathfrak{M}$. Let supp $v_{\mathfrak{n}}$ denote the support of the measure $v_{\mathfrak{n}}$

1. There exists a unique one-to-one continuous map $T^{\prime}: \operatorname{supp} v_{\mathfrak{M}} \rightarrow \mathscr{G}_{\Phi}$ which maps $\omega \in \operatorname{supp} v_{\mathfrak{R}}$ onto $T^{\prime} \omega \in \mathscr{G}_{\Phi}$ with

$$
\left(T^{\prime} \omega\right)(f)=\omega(T(f))
$$

for all $f \in C_{0}(\Omega)$. Let $v_{\mathfrak{N}}^{*}$ be the measure on $E_{C(\Omega)}$ which is the image measure of $v_{n}$ under the map $T^{\prime}$.

2. The measure $v_{\mathfrak{R}}^{*}$ is the orthogonal on $E_{C(\Omega)}$ which corresponds to $\mathfrak{N}$. In particular

$$
\mu=\int_{E_{C}(\Omega)} v_{\mathfrak{M}}^{*}\left(d \mu^{\prime}\right) \mu^{\prime}
$$

3. The support of $v_{\cdots}^{*}$ consists of $\theta_{0}$-invariant Gibbs states. There exists a subset of $E_{C(\Omega)}$ with $v^{*}$ measure 1 , which consists of states that have the Markov property with respect to $P_{0}$.

Proof. 1. and 2. Let $\tilde{\mathfrak{N}}$ be a finite dimensional subalgebra of $3_{\omega}$. Let $\left\{Q_{i}\right\}_{i=1, \ldots, n}$ be the maximal set of mutually orthogonal nonzero projections in $\tilde{\mathfrak{R}}$. Then the orthogonal measure $v_{\tilde{\mathfrak{N}}}$ on $E_{\mathfrak{Q}}$ corresponding to $\tilde{\mathfrak{N}}$ is given by

$$
v_{\tilde{\mathfrak{R}}}=\sum_{i=1}^{n} \omega_{Q_{1}}(1) \delta_{\omega_{\mathrm{i}}},
$$

where $\delta_{\omega_{i}}$ is the Dirac measure at $\omega_{\imath} \in E_{\mathrm{yI}}$ with $\omega_{i}$ given by

$$
\omega_{i}(\sigma)=\frac{1}{\omega_{Q_{1}}(\mathbb{1})} \omega_{Q_{i}}(\sigma)
$$

for $\sigma \in \mathfrak{U}$ and $i=1, \ldots, n$. Thus the map $T^{\prime}$ is readily defined on the support of $v_{\tilde{\mathfrak{N}}^{\prime}}$ which is $\left\{\omega_{i} \mid i=1, \ldots, n\right\}$. Explicitly,

$$
T^{\prime} \omega_{i}=\frac{1}{Q_{i}(1)} \mu_{Q_{i}} .
$$

Therefore

$$
v_{\mathfrak{N}}^{*}=\sum_{i=1}^{n} \omega_{Q_{i}}(\uparrow) \delta_{\mu_{i}},
$$

where $\delta_{\mu_{i}}$ is the Dirac measure at $\mu_{i} \in E_{C(\Omega)}$ with $\mu_{i}=1 / \omega_{Q_{i}}(1) \mu_{Q_{i}}$. Therefore the statements under 1 . and 2 . hold with $\mathfrak{R}$ replaced by $\tilde{\mathfrak{N}}$. But $v_{\mathfrak{M}}$ is the vague limit of the net $\left\{v_{\tilde{\mathfrak{R}}}\right\}$ with $\tilde{\mathfrak{N}}$ finite von Neumann algebras in $\mathfrak{N}$, where the ordering of the net is given by inclusion of the von Neumann algebras. Similarly the orthogonal measure on $E_{C(\Omega)}$ which corresponds to $\mathfrak{N}$ is the vague limit of the net $\left\{v_{\mathfrak{N}}^{*}\right\}$ 
with $\tilde{\mathfrak{N}}$ finite von Neumann algebras in $\mathfrak{N}$. It follows that the statements 1 . and 2. hold for $\mathfrak{N}$.

3. The fact that the support of $v_{\mathfrak{N}}^{*}$ is contained in the set of $\theta_{0}$-invariant states on $E_{C(\Omega)}$, is obvious. The Hilbert space $\mathfrak{S}_{\omega}$ can be written as a direct integral Hilbert space as

Then

$$
\mathfrak{H}_{\omega}=\int_{E_{\mathscr{Q}}}^{\oplus} v_{\mathfrak{N}}\left(d \omega^{\prime}\right) \mathfrak{H}_{\omega^{\prime}}
$$

$$
\pi_{\omega}=\int^{\oplus} v_{\Re}\left(d \omega^{\prime}\right) \pi_{\omega^{\prime}} \quad \text { and } \quad \Omega_{\omega}=\int^{\oplus} v_{\Re}\left(d \omega^{\prime}\right) \Omega_{\omega^{\prime}}
$$

Let $\left\{\psi_{n}\right\}_{n \in \mathbb{N}}$ be a fundamental sequence of fields. In particular $\left\{\psi_{n}\left(\omega^{\prime}\right)\right\}_{n \in \mathbb{N}}$ is a dense sequence in $\mathfrak{H}_{\omega^{\prime}}$. By Lemma 3 one has from the Markov property of $\mu$ that $\pi_{\omega}\left(\mathfrak{I}_{0}^{\text {diag }}\right) \Omega_{\omega}$ is dense in $\mathfrak{H}_{\omega}$. Therefore, for each $n \in \mathbb{N}$, there exists a sequence $\left\{\sigma_{n, k}\right\}_{k \in \mathbb{N}}$ in $\mathfrak{U}_{0}^{\text {diag }}$ such that

$$
\pi_{\omega^{\prime}}\left(\sigma_{n, k}\right) \Omega_{\omega^{\prime}} \rightarrow \psi_{n}\left(\omega^{\prime}\right)
$$

in $\mathfrak{H}_{\omega^{\prime}}$ for $v_{\mathfrak{P}^{\prime}}$-almost all $\omega^{\prime}$. Thus one sees that $\pi_{\omega^{\prime}}\left(\mathfrak{U}_{0}^{\text {diag }}\right) \Omega_{\omega^{\prime}}$ is dense in $\mathfrak{H}_{\omega^{\prime}}$ for $v_{\mathfrak{M}}$-almost all $\omega^{\prime}$. Another application of Lemma 3 then gives that $T^{\prime} \omega^{\prime}$ has the Markov property with respect to $P_{0}$.

Let $G$ be a subgroup of $\mathbb{Z}^{d}$. The set $E_{C(\Omega)}^{G}$ is a simplex. Its set of extremal point is denoted by $\operatorname{Ext}\left(E_{C(\Omega)}^{G}\right)$. Since $C(\Omega)$ is separable, $\operatorname{Ext}\left(E_{C(\Omega)}^{G}\right)$ is a Baire set. If $N \in \mathbb{N}$ define a map, $A_{N}^{G}: C(\Omega) \rightarrow C(\Omega)$ which maps $f \in C(\Omega)$ onto $A_{N}^{G} f \in C(\Omega)$, where $A_{N}^{G} f$ is defined by

$$
A_{N}^{G} f=\frac{1}{\left|\Lambda_{N} \cap G\right|} \sum_{a \in A_{N} \cap G} \tau^{(a)} f .
$$

Then $\mu \in \operatorname{Ext}\left(E_{(\Omega)}^{G}\right)$ if and only if for all $f, g \in C_{0}(\Omega)$,

$$
\lim _{N \rightarrow \infty}\left|\mu\left(g\left(A_{N}^{G} f\right)\right)-\mu(g) \mu(f)\right|=0 .
$$

Let $H$ be a nontrivial group contained in $\mathbb{Z}^{d-1}$. The set $E_{\mathfrak{U}}^{H}$ is a simplex. The set of its extremal points is denoted by $\operatorname{Ext}\left(E_{\mathfrak{U}}^{H}\right)$. Since $\mathfrak{U}$ is separable, $E_{\mathfrak{Q}}^{H}$ is a Baire set.

If $N \in \mathbb{N}$, define a map $A_{N}^{H}: \mathfrak{A} \rightarrow \mathfrak{A}$ which maps $\sigma \in \mathfrak{A}$ onto $A_{N}^{H}(\sigma) \in \mathfrak{A}$, where $A_{N}^{H}(\sigma)$ is given by

$$
A_{N}^{H}(\sigma)=\frac{1}{\left|\Lambda_{n} \cap H\right|} \sum_{a \in \Lambda_{N} \cap H} \tau^{(a)}(\sigma) .
$$

Then $\omega \in \operatorname{Ext}\left(E_{\mathscr{U}}^{H}\right)$ if and only if for all $\sigma_{1}, \sigma_{2} \in \mathfrak{N}_{0^{\prime}}$

$$
\lim _{N \rightarrow \infty}\left|\omega\left(\sigma_{2} A_{N}^{H}\left(\sigma_{1}\right)\right)-\omega\left(\sigma_{2}\right) \omega\left(\sigma_{1}\right)\right|=0 .
$$

Let $(\mu, \omega)$ be a canonical state pair. Let $H \subseteq \mathbb{Z}_{\mathrm{ev}}^{d-1}$ be a nontrivial subgroup. Then $\mu \in E_{C(\Omega)}^{H}$ if and only if $\omega \in E_{\mathfrak{q}}^{H}$. Moreover $\mu \in \operatorname{Ext}\left(E_{C(\Omega)}^{H}\right)$ if and only if 
$\omega \in \operatorname{Ext}\left(E_{\mathfrak{g}}^{H}\right)$. This is most easily seen by observing that for $a \in \mathbb{Z}_{\mathrm{ev}}^{d}$ we have

$$
T\left(f\left(\tau^{(a)} g\right)\right)=T(f) \tau^{(a)} T(g)
$$

as soon as $\min _{i=1, \ldots . d-1}\left|a_{i}\right|$ is larger than some constant (which depends on $f$ and $g$ ) and then using the characterization of $H$-extremality as stated above. Now assume furthermore that $\mu$ has the Markov property with respect to $P_{0}$. Write $\mathfrak{N}=\mathfrak{R}_{\omega}(H)$. Let $v_{\mathfrak{M}}$ be the orthogonal measure on $E_{\mathfrak{Y}}$ corresponding to $\mathfrak{N}$ and $v_{\mathfrak{M}}^{*}$ the orthogonal measure on $E_{C(\Omega)}$ corresponding to $\mathfrak{i}$. By standard theory $v_{\mathfrak{R}}\left(\operatorname{Ext}\left(E_{\mathfrak{l}}^{H}\right)\right)=1$. Therefore by the foregoing remarks and Lemma 6,

$$
v_{\mathfrak{R}}^{*}\left(\operatorname{Ext}\left(E_{C(\Omega)}^{H}\right)\right)=1 .
$$

Therefore $v_{\mathfrak{N}}^{*}$ is the measure on $E_{C(\Omega)}^{H}$ which gives the $H$-ergodic decomposition of $\mu$, i.e., $v_{\mathfrak{R}}^{*}$ is the unique maximal measure on $E_{C(\Omega)}^{H}$ which represents $\mu$. Write $\mathfrak{S}_{\omega}$, as the direct integral

$$
\mathfrak{H}_{\omega}=\int^{\oplus} v_{\mathfrak{H}}\left(d \omega^{\prime}\right) \mathfrak{S}_{\omega^{\prime}}
$$

Assume $P_{\infty}^{(2 n)} \geqq 0$. Then, since $P_{\infty}^{(2 n)} \in \mathfrak{M}^{\prime}$, this operator is decomposable. Hence one can write

$$
\begin{aligned}
P_{\infty}^{(2 n)} & =\int^{\oplus} v_{\mathfrak{M}}\left(d \omega^{\prime}\right) P_{\infty, \omega^{\prime}}^{(2 n)} \text {. Now note, if } \sigma \in \mathfrak{P}_{0^{\prime}} \int^{\oplus} v_{\mathfrak{M}}\left(d \omega^{\prime}\right) \pi_{\omega^{\prime}}\left(x^{(2 n)}(\sigma)\right) \Omega_{\omega^{\prime}} \\
& =\pi_{\omega}\left(\alpha^{(2 n)}(\sigma)\right) \Omega_{\omega^{\prime}}=P_{\infty}^{(2 n)} \pi_{\omega}(\sigma) \Omega_{\omega}=\int^{\oplus} v_{\Re}\left(d \omega^{\prime}\right) P_{\infty, \omega}^{(2 n)} \pi_{\omega}(\sigma) \Omega_{\omega^{\prime}} .
\end{aligned}
$$

Hence

$$
P_{\infty, \omega \omega^{\prime}}^{(2 n)}, \pi_{\omega^{\prime}}(\sigma) \Omega_{\omega^{\prime}}=\pi_{\omega)^{\prime}}\left(\alpha^{(2 n)}(\sigma)\right) \Omega_{\omega^{\prime}}
$$

for $v_{\mathfrak{R}}$-almost all $\omega^{\prime}$. Therefore for $v_{\mathfrak{N}}$-almost all $\omega^{\prime}$,

$$
\left(\omega^{\prime} \circ \alpha^{(2 n)}=\omega\right)^{\prime} \text { on } \quad \mathfrak{U}_{0} .
$$

This implies that for $v_{*-a}^{*}$-almost all $\mu^{\prime} \in E_{C(\Omega)}^{H}$, the state $\mu^{\prime}$ is invariant under $\tau_{0}^{2 n}$.

Corollary 2. Take $\tilde{\mu} \in \mathscr{G}_{\Phi} \cap E_{C(\Omega)}^{\mathbb{Z}_{\mathrm{e}}^{d}}$. Then $\tilde{\mu}$ is $\theta_{0}$-invariant.

Proof. It is sufficient to prove this under the further assumption that $\tilde{\mu} \in \operatorname{Ext}\left(E_{C(\Omega)}^{Z_{\mathrm{ev}}^{d}}\right)$. Consider $\mu=\frac{1}{2}\left(\tilde{\mu}+\tilde{\mu} \circ \theta_{0}\right)$. Assume, ad absurdum, that $\tilde{\mu} \neq \tilde{\mu} \circ \theta_{0}$. Then $\tilde{\mu}$ is disjoint from $\tilde{\mu}^{\circ} \theta_{0}$. Let $H \subseteq \mathbb{Z}_{\mathrm{ev}}^{d-1}$ be a nontrivial group. Then there exists $S \subset E_{C(\Omega)}^{H}$ such that

$$
\tilde{\mu}=\frac{1}{v^{*}(S)} \int_{S} v^{*}\left(d \mu^{\prime}\right) \mu^{\prime}
$$

where $v^{*}$ is the unique maximal measure on $E_{C(\Omega)}^{H}$ which represents $\mu$. Now $\mu$ is a $\theta_{0}$-invariant and $\mathbb{Z}_{\mathrm{ev}}^{d}$-invariant Gibbs state. Therefore $\mu$ has the Markov property with respect to $P_{0}$ (Corollary 1$)$. Let $(\mu, \omega)$ be the canonical state pair which can be constructed from $\mu$. Write $\mathfrak{N}=\mathfrak{R}_{\omega}(H)$. Then

$$
v^{*}=v_{\mathfrak{N}}^{*},
$$


where notation is used which has been introduced just prior to Corollary 2. But $v_{\mathfrak{M}}^{*}$ is concentrated on $\theta_{0}$-invariant states by Lemma 6 , part 3 . Hence by (13) it follows that $\tilde{\mu}$ is $\theta_{0}$-invariant. This is in contradiction with $\tilde{\mu} \neq \tilde{\mu}^{\circ} \theta_{0}$. Therefore $\tilde{\mu}=\tilde{\mu} \circ \theta_{0}$.

From Corollary 2 one obtains that $\mathbb{Z}_{\mathrm{ev}}^{d}$-invariant Gibbs states have the following properties:

1. $\theta_{k}$-invariance for each $k \in \mathbb{Z}$,

2. the Markov property with respect to $P_{k}$ for each $k \in \mathbb{Z}$,

3. $\theta_{k}$-reflection positivity for each $k \in \mathbb{Z}$.

If $j \in\{0, \ldots, d-1\}$ denote by $G_{j}^{(2)}$ the subgroup of $\mathbb{Z}_{\mathrm{ev}}^{d}$ generated by $a \in \mathbb{Z}^{d}$ where $a_{i}=2 \delta_{i, j}$ for $i=0, \ldots, d-1$.

Corollary 3. Take $\mu \in \mathscr{G}_{\Phi} \cap \operatorname{Ext}\left(E_{C(\Omega)}^{\mathbb{Z}_{\mathrm{ev}}^{d}}\right)$. Then $\mu \in \operatorname{Ext}\left(E_{C(\Omega)}^{G_{2}^{(2)}}\right)$ for all $j=0, \ldots, d-1$.

Proof. Consider the group $G_{1}^{(2)}$ and let $v^{*}$ be the unique maximal measure on $E_{C(\Omega)}^{G_{1}^{(2)}}$ which represents $\mu$. Then the support of $v^{*}$ is contained in the set $E_{C(\Omega)}^{G_{0}^{(2)}}$ by the remarks made prior to Corollary 2. (Note that $P_{\infty}^{(2)} \geqslant 0$, since $\mu$ is $\theta_{1}$-reflection positive.) But the 0-direction plays no particular role to the 1-direction: one could have done a transfer-matrix formalism in the $k$-direction, where $k \in\{2, \ldots, d-1\}$, with the result that the support of $v^{*}$ is contained in $E_{C(\Omega)}^{G_{k}^{(2)}}$. Hence the support of $v^{*}$ is contained in $E_{C(\Omega)}^{\mathbb{Z}_{\mathrm{ev}}^{d}}$. Since $\mu \in \operatorname{Ext}\left(E_{C(\Omega)}^{\mathbb{Z}_{\mathrm{ev}}^{d}}\right)$, one obtains $v^{*}=\delta_{\mu}$, where $\delta_{\mu}$ is the Dirac measure at $\mu \in E_{C(\Omega)}$. Hence $\mu \in \operatorname{Ext}\left(E_{C(\Omega)}^{G_{1}^{(2)}}\right)$. But the 1-direction plays no special role. Therefore $\mu \in \operatorname{Ext}\left(E_{C(\Omega)}^{G_{j}^{(2)}}\right)$ for each $j \in\{0, \ldots, d-1\}$.

Corollary 3 is strengthened in part 2 of Theorem 3 .

Theorem 3. Let $\mu \in \mathscr{G}_{\Phi} \cap \operatorname{Ext}\left(E_{C(\Omega)}^{\mathbb{Z}_{\mathrm{Z}}^{d}}\right)$. Then:

1. the state $\mu$ is strongly clustering for $\mathbb{Z}_{\mathrm{ev}}^{d}$, i.e., for all $f, g \in C_{0}(\Omega)$ one has

$$
\lim _{\substack{a \in \mathbb{Z}_{\text {ev }}^{d} \\|a| \rightarrow \infty}}\left|\mu\left(g\left(\tau^{(a)} f\right)\right)-\mu(g) \mu(f)\right|=0,
$$

where $\|\quad\|$ is the standard norm of $\mathbb{Z}^{d} \subset \mathbb{R}^{d}$;

2. for each $j \in\{0, \ldots, d-1\}$ the state $\mu$ is clustering of all orders for $G_{j}^{(2)}$, i.e., for each $n \in \mathbb{N}$ and $f_{1}, \ldots, f_{n} \in C_{0}(\Omega)$ one has

$$
\lim _{\substack{\left(k_{1}, \ldots, k_{n}\right) \in \mathbb{Z}^{n} \\ \ln \left\{\left\{\mid k_{i}-k_{,}\right\} \mid l \neq j \text { and } \\ 1, j \in\{1, \quad, n\}\right\} \rightarrow \infty}}\left|\mu\left(\left(\tau_{j}^{2 k_{1}} f_{j}\right) \cdots\left(\tau_{j}^{2 k_{n}} f_{n}\right)\right)-\mu\left(f_{1}\right) \cdots \mu\left(f_{n}\right)\right|=0 .
$$

Proof. From the fact that $\mu \in \operatorname{Ext}\left(E_{C(\Omega)}^{G_{0}^{(2)}}\right)$ one obtains that the sequence

$$
\left\{\frac{1}{N} \sum_{m=0}^{N-1} P_{\infty}^{(2)^{m}}\right\}_{N \in \mathbb{N}}
$$

converges weakly to $P_{\Omega_{\omega}}$, where $P_{\Omega_{\omega}}$ is the orthogonal projection on $\mathfrak{H}_{\omega}$ with range $\mathbb{C} \Omega_{\omega}$. (This is a restatement of the cluster property characterizing the state $\mu$ as an element of $\operatorname{Ext}\left(E_{C(\Omega)}^{G_{0}^{(2)}}\right)$ ). Since $P_{\infty}^{(2)}$ is a positive contraction, the sequence $\left\{P_{\infty}^{(2)^{m}}\right\}_{m \in \mathbb{N}}$ has $P_{\Omega_{\omega}}$ as strong limit. 
1. For $a \in \mathbb{Z}_{\mathrm{ev}}^{d}$ write

$$
t(a)=\mu\left(g\left(\tau^{(a)} f\right)\right)
$$

Let $\left\{t\left(a^{(k)}\right)\right\}_{k \in \mathbb{N}}$ be a convergent subsequence of $\{t(a)\}_{a \in \mathbb{Z}_{\mathrm{ev}}^{\mathrm{d}}}$. Then at least one of the following statements $a$. and $b$. is true:

a. There exists $j \in\{0, \ldots, d-1\}$ and a subsequence $\left\{t\left(b^{(l)}\right)\right\}_{l \in \mathbb{N}}$ of $\left\{t\left(a^{(k)}\right)\right\}_{k \in \mathbb{N}}$ such that $b_{j}^{(l)} \rightarrow-\infty$ as $l$ tends to infinity.

b. There exists $j \in\{0, \ldots, d-1\}$ and a subsequence $\left\{t\left(c^{(l)}\right)\right\}_{l \in \mathbb{N}}$ of $\left\{t\left(a^{(k)}\right)\right\}_{k \in \mathbb{N}}$ such that $c_{j}^{(l)} \rightarrow \infty$ as $l$ tends to infinity.

Assume that statements a. is true. There is no loss of generality in assuming that $j=0$. (Otherwise one should employ a transfer-matrix formalism in the $j$-direction in the following.) Also without loss of generality we may assume that $g \in C_{\mathbb{Z}_{0,-}^{d}}$. Then

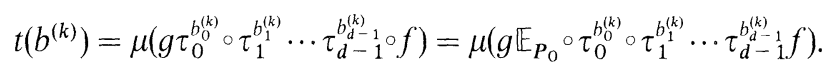

By the invariance of $\mu$ under $\mathbb{Z}_{\mathrm{ev}}^{d}$ and the strong convergence of $P_{\infty}^{(2)^{b_{0 / 2}}}=$ $\mathbb{E}_{P_{0}} \circ \tau_{0}^{b_{0}^{(k)}}$ to $P_{\Omega_{\omega}}$ one obtains that

$$
\lim _{k} t\left(b^{(k)}\right)=\mu(g) \mu(f) .
$$

This proves case a. However by reflectional invariance case $b$. is easily reduced to case a. So part 1 of the theorem has been proven.

2. There is no loss of generality in assuming $j=0$. If $g_{1}, \ldots, g_{n} \in C_{0}(\Omega)$, consider the sequence $\{s(l)\}$ where $s(l)=\mu\left(\left(\tau_{0}^{2 l_{1}} g_{1}\right) \cdots\left(\tau_{0}^{2 l_{n}} g_{n}\right)\right)$. Using that the strong limit of $\left\{P_{\infty}^{(2)^{m}}\right\}_{m \in \mathbb{N}}$ is $P_{\Omega}$, one easily sees

Define

$$
\lim _{\substack{l_{l+1}-l_{i} \rightarrow \infty \\ \text { for } i=1, \ldots, n-1}} s(l)=\mu\left(g_{1}\right) \cdots \mu\left(g_{n}\right)
$$

$$
t(k)=\mu\left(\left(\tau_{0}^{2 k_{1}} f_{1}\right) \cdots\left(\tau_{0}^{2 k_{n}} f_{n}\right)\right),
$$

where $k \in \mathbb{Z}^{n}$. Then it is readily seen that each convergent subsequence of $\{t(k)\}_{k \in \mathbb{Z}^{n}}$ contains a subsequence of the type $\{s(l)\}_{l \in \mathbb{Z}^{n}}$ with $\left(g_{1}, \ldots, g_{n}\right)$ a permutation of $\left(f_{1}, \ldots, f_{n}\right)$. Therefore each convergent subsequence of $\{t(k)\}_{k \in \mathbb{Z}^{n}}$ has the limit $\mu\left(f_{1}\right) \cdots \mu\left(f_{n}\right)$. Hence $\{t(k)\}_{k \in \mathbb{Z}^{n}}$ itself has this limit.

Corollary 4. Let $\mu \in \mathscr{G}_{\Phi}$ be invariant under a subgroup $G \subseteq \mathbb{Z}_{\mathrm{ev}}^{d}$ with finite index in $\mathbb{Z}_{\mathrm{ev}}^{d}$. Then $\mu$ is $\mathbb{Z}_{\mathrm{ev}}^{d}$-invariant.

Proof. Without losing generality one may assume that $\mu \in \operatorname{Ext}\left(E_{C(\Omega)}^{G}\right)$. Choose $M \subset \mathbb{Z}_{\mathrm{ev}}^{d}$ such that $\{a+M\}_{a \in G}$ is a partition of $\mathbb{Z}_{\mathrm{ev}}^{d}$ and choose $\tilde{M} \subset M$ as a maximal set in $M$ with the property that $\mu^{\circ} \tau^{(x)} \neq \mu^{\circ} \tau^{(y)}$ for all $x, y \in \tilde{M}$ with $x \neq y$. Consider

$$
\tilde{\mu}=\frac{1}{|\tilde{M}|} \sum_{x \in \tilde{M}} \mu \circ \tau^{(x)} .
$$


Then $\tilde{\mu}$ is $\mathbb{Z}_{\mathrm{ev}}^{d}$-invariant and moreover $\tilde{\mu} \in \operatorname{Ext}\left(E_{C(\Omega)}^{Z_{\mathrm{ev}}^{d}}\right)$. By Theorem 3, part 1. the state $\tilde{\mu}$ is in $\operatorname{Ext}\left(E_{C(\Omega)}^{G}\right)$. But (14) establishes a decomposition of $\tilde{\mu}$ into $G$-invariant state. It follows that $|\tilde{M}|=1$, i.e.,

$$
\mu \circ \tau^{(x)}=\mu
$$

for all $x \in M$. Hence $\mu$ is $\mathbb{Z}_{\mathrm{ev}}^{d}$-invariant.

\section{Two-Dimensional Ising Model}

Consider the two-dimensional Ising model in zero external field (and with nonzero coupling constants in both directions of the lattice $\mathbb{Z}^{2}$ ). First suppose that the temperature is below the critical temperature. Let $\mu$ be a Gibbs state with zero magnetization. Then by Aizenman's result [15], $\mu=\frac{1}{2}\left(\mu_{+}+\mu_{-}\right)$, where $\mu_{+}$and $\mu_{-}$ are extremal Gibbs states. Moreover the states $\mu_{+}$and $\mu_{-}$are $\mathbb{Z}^{d}$-invariant.

Let $\omega$ be the state on $\mathfrak{A}$ associated with $\mu:(\mu, \omega)$ is a canonical state pair. Then there exists a projection $Q \in 3_{\omega}$ such that

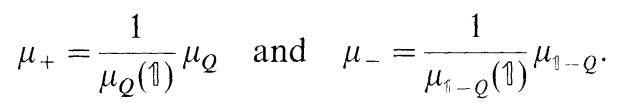

Write $\omega_{+}=\left(1 / \omega_{Q}(1)\right) \omega_{Q}$ and $\omega_{-}=\left(1 / \omega_{\vartheta_{-}}(1)\right) \omega_{Q}$. Then $\left(\mu_{+}, \omega_{+}\right)$and $\left(\mu_{-}, \omega_{-}\right)$are canonical state pairs. And $\omega=\frac{1}{2}\left(\omega_{+}+\omega_{-}\right)$is a decomposition of $\omega$ in disjoint states. Furthermore $\omega_{+}$and $\omega_{-}$are irreducible states since $\mu_{+}$and $\mu_{-}$are extremal Gibbs states. (If $\omega_{+}$or $\omega_{-}$were not irreducible one could by Lemma 6 construct a nontrivial decomposition of $\mu_{+}$or $\mu_{-}$into other Gibbs states.)

Now consider the case where the temperature is equal or greater than the critical temperature. Then there is a unique Gibbs state $\mu$. Hence the state $\omega$ on $\mathfrak{A}$ associated with $\mu$ is irreducible. In this way one has re-obtained the main result of the paper [7] by Araki and Evans.

Acknowledgement. The author gratefully acknowledges several discussions with $\mathrm{M}$. Winnink.

\section{References}

1. Malyshev, V. A.: Cluster expansions in lattice models of statistical physics and the quantum theory of fields. Russ. Math. Surv. 35, 1-62 (1980)

2. Goldstein, S.: Remarks on the global Markov property. Commun. Math. Phys. 74, 223-234 (1980)

3. Fredenhagen, K.: On the existence of the real-time evolution in Euclidean lattice gauge theories. Commun. Math. Phys. 101, 579-587 (1985)

4. Pirogov, S. A.: States associated with the two-dimensional Ising model. Theor. Math. Phys. 11, 614-617 (1972)

5. Lewis, J. T., Sisson, P. N. M.: A $C^{*}$-algebra of the two-dimensional Ising model. Commun. Math. Phys. 44, 279-292 (1975)

6. Lewis, J. T., Winnink, M.: The Ising model phase transition and the index of states on the Clifford algebra. In: Random fields. Fritz, J., Lebowitz, J. L., Szasz, D. (eds.). pp.671-679. Amsterdam: North Holland 1981

7. Araki, H., Evans, D. E.: On a $C^{*}$-algebra approach to phase transition in the two-dimensional Ising model. Commun. Math. Phys. 91, 489-503 (1983) 
8. Nelson, F.: Probability theory and Euclidean field theory. In: Constructive quantum field theory. Velo, G., Wightman, A. (eds.). pp. 94-124. Berlin. Heidelberg, New York: Sprınger 1973

9. Osterwalder, K., Schrader, R.: Axioms for Euclidean Green's functions. Commun. Math. Phys. 31, 83-112 (1973)

10. Schor, R. S.: The particle structure of $v$-dimensional Ising models at low temperatures. Commun. Math. Phys. 59, 213-233 (1978)

11. Kuik, R.: Global Markov property for equilibrium states which are determined by correlations in a strip. Commun. Math. Phys. (in press)

12. Simon, B.: In: The $P(\Phi)_{2}$ Euclidean (quantum) field theory. Princeton, New Jersey: Princeton University Press 1974

13. Bratteli, O., Robinson, D. W.: Operator algebras and quantum statistical mechanics I. Berlın, Heidelberg, New York: Springer 1979

14. Georgii, H. O.: Two remarks on extremal equilibrium states. Commun. Math. Phys. 32, 107-118 (1973)

15. Aizenman, M.: Translation invariance and instability of phase coexistence in the two-dimensional Ising system. Commun. Math. Phys. 73, 83-94 (1980)

16. Fisher, M. F., Selke, W.: Infinitely many commensurate phases in a simple Ising model. Phys. Rev. Lett. 44, 1502-1505 (1980)

Communicated by M. Aizenman

Received March 11, 1987; in revised form October 14, 1987 